\title{
I Zw 18 as morphological paradigm for rapidly assembling high-z galaxies
}

\author{
P. Papaderos ${ }^{1,2}$ and G. Östlin ${ }^{2}$ \\ ${ }^{1}$ Centro de Astrofísica and Faculdade de Ciências, Universidade do Porto, Rua das Estrelas, 4150-762 Porto, Portugal \\ e-mail: papaderos@astro.up.pt \\ 2 Department of Astronomy, Oskar Klein Centre, Stockholm University, 10691 Stockholm, Sweden
}

Received 23 June 2011 / Accepted 18 October 2011

\section{ABSTRACT}

\begin{abstract}
Context. I Zw 18, ever since regarded as the prototypical blue compact dwarf (BCD) galaxy, is, quite ironically, the most atypical BCD known. This is because its large low-surface brightness (LSB) envelope is not due to an old underlying stellar host, as invariably is the case for typical BCDs, but is entirely due to extended nebular emission.

Aims. Our goal is to explore I $\mathrm{Zw} 18$ and its detached $\mathrm{C}$ component I Zw $18 \mathrm{C}$ down to an unprecedently faint surface brightness $\mu$ $\left(\mathrm{mag} / \square^{\prime \prime}\right)$ level in order to gain further insight into the structural properties and evolutionary history of this enigmatic galaxy pair. Methods. We present a photometric analysis of the entire set of archival HST ACS $V, R$ and $I$ band data for I Zw 18.

Results. Radial color profiles for I Zw $18 \mathrm{C}$ reveal blue and practically constant colors $(0 \pm 0.05)$ down to $\mu \sim 27.6$, and a previously undisclosed, slightly redder $(V-I \approx 0.2)$, stellar population in its extreme periphery $(\mu \sim 29)$. We argue that stellar diffusion over $\tau \sim 10^{8} \mathrm{yr}$ and the associated stellar mass filtering effect can consistently account for the observed properties of the stellar component in the outskirts of $\mathrm{I} \mathrm{Zw} 18 \mathrm{C}$. This process, in combination with propagating star formation with a mean velocity of $\sim 20 \mathrm{~km} \mathrm{~s} \mathrm{~s}^{-1}$ can reproduce all essential characteristics of I Zw $18 \mathrm{C}$ within $\sim \tau$. An extremely faint substrate of older stars cannot be ruled out but does not need to be postulated. As for I Zw 18, we find that nebular emission (ne) extends out to $\sim 16$ stellar scale lengths, shows a nearly exponential outer profile, and provides at least one third of the total optical emission. Nebular emission dominates already at $\mu \sim 23.5$, as evident from e.g. the uniform and extremely blue $(V-I \approx-1, R-I \approx-1.4)$ colors of the LSB envelope of I Zw 18.

Conclusions. The case of I $\mathrm{Zw} 18$ suggests caution in studies of distant galaxies in dominant stages of their evolution, rapidly assembling their stellar mass at high specific star formation rates (SSFRs). It calls attention to the fact of ne not necessarily being cospatial with the underlying ionizing and non-ionizing stellar background. It also does not have to scale with the background surface density. The prodigious energetic output during dominant phases of galaxy evolution may result in large exponential ne envelopes, extending much beyond the still compact stellar component, just like in I Zw 18. Therefore, the morphological paradigm of I Zw 18, while probably unique in the nearby Universe, may be ubiquitous among high-SSFR galaxies at high redshift. Using I Zw 18 as reference, we show that extended ne may introduce substantial observational biases and affect several of the commonly studied fundamental galaxy relations. Among others, we show that the surface brightness profiles of distant morphological analogs to I Zw 18 may be barely distinguishable from Sérsic profiles with an exponent $2 \lesssim \eta \lesssim 5$, thus mimicking the profiles of massive galaxy spheroids.
\end{abstract}

Key words. galaxies: structure - galaxies: evolution - galaxies: dwarf - galaxies: high-redshift - galaxies: halos - galaxies: starburst

\section{Introduction}

Even four decades after its discovery (Sargent \& Searle 1970), the blue compact dwarf (BCD) galaxy I Zw 18 continues to attract considerable interest and feed intense debates in extragalactic research. Its low oxygen abundance (Searle \& Sargent 1972), established in numerous subsequent studies (Lequeux et al. 1979; French 1980; Kinman \& Davidson 1981; Pagel et al. 1992; Skillman \& Kennicutt 1993; Martin 1996; Vílchez \& Iglesias-Páramo 1998; Izotov \& Thuan 1998a,b; Izotov et al. 1999 b, among others) to be $12+\log (\mathrm{O} / \mathrm{H}) \approx 7.2$, makes it the third most metal-poor star-forming (SF) galaxy in the nearby Universe, after SBS 0335-052 W (Izotov et al. 2005; Papaderos et al. 2006a; Izotov et al. 2009) and DDO68 (Pustilnik et al. 2005; Izotov \& Thuan 2007). Despite a meanwhile long record of extremely metal-poor BCDs $(12+\log (\mathrm{O} / \mathrm{H}) \leqslant 7.6$; hereafter XBCDs) discovered in the recent years (see e.g. Papaderos et al. 2008; Guseva et al. 2009, for a review), I Zw 18 remains the unconquered prototypical example of this enigmatic galaxy class.
I Zw 18 was originally described by Zwicky (1966) as a pair of compact galaxies, later on recognized to be SF regions within the same galaxy, the brighter northwestern and the fainter southeastern component, referred to as regions NW and SE, which are separated by $\approx 6^{\prime \prime}$ (cf. Fig. 1). Subsequent work has shown that these regions are embedded within an extended, lowsurface brightness (LSB) envelope (Davidson et al. 1989; Dufour \& Hester 1990; Östlin et al. 1996; Dufour et al. 1996a; Martin 1996), whose rich filamentary substructure was impressively revealed with the advent of the HST (Hunter \& Thronson 1995; Dufour et al. 1996b). That nebular emission (hereafter ne) is very strong in the central part of I Zw 18 and its northwestern supershell was spectroscopically documented early on. For example, Izotov et al. (2001a) have shown, on the basis of deep long slit spectroscopy, that the equivalent width $(E W)$ of the $\mathrm{H} \alpha$ emission line rises to $1700 \AA$ northwest of region NW and that ne is present as far away as $15^{\prime \prime}$ from it (regions labeled " $\mathrm{H} \alpha$ arc" and "Loop" in Fig. 1). The $E W(\mathrm{H} \alpha)$ morphology of I $\mathrm{Zw} 18$ was first studied with high-resolution, ground-based imagery 


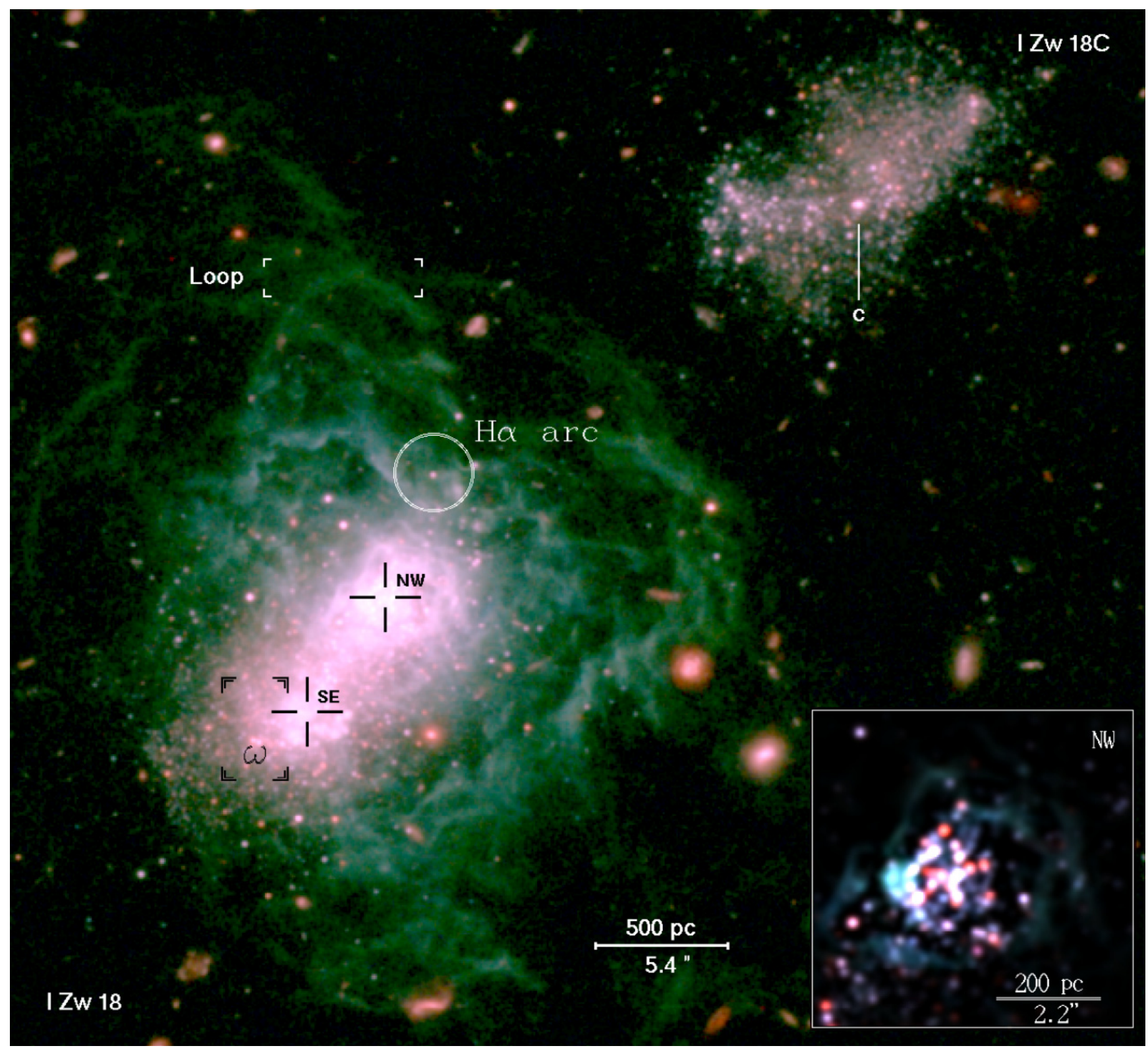

Fig. 1. Three-color composite image of I Zw 18 and I Zw $18 \mathrm{C}$, combining HST ACS data in $V, R$, and $I$ (blue, green, and red channels, respectively). The position of the SF regions NW and SE is indicated by crosses. The regions labeled "Loop" and "H $\alpha$ arc" were studied through deep Keck II long slit spectroscopy by Izotov et al. (2001a). The region $\omega$ at the southeastern tip of I Zw 18 shows comparatively weak nebular emission, its colors therefore allow meaningful constraints to be placed on the age of the stellar component (Papaderos et al. 2002). In the magnified version of region NW (inset), combining the unsharp masked images $I_{\mathrm{c}}, R_{\mathrm{c}}$, and $V_{\mathrm{c}}$ (see Sect. 3 for details), about 30 point sources, surrounded by a complex network of ionized gas shells are discernible. The irregular blue galaxy I Zw $18 \mathrm{C}$ is located $\sim 22^{\prime \prime}$ northwest of region $\mathrm{NW}(\approx 2 \mathrm{kpc}$ at the assumed distance of $19 \mathrm{Mpc}$ to I Zw 18). It shows faint nebular emission in its bluer southeastern tip and central star cluster complex C (Izotov et al. 2001a). North is at the top and east to the left.

by Östlin et al. (1996) who described a horseshoe-shaped rim of intense $(E W(\mathrm{H} \alpha) \simeq 1500 \AA)$ ne encompassing region NW. This conspicuous $E W$ pattern was later confirmed through HST WFPC2 data (Papaderos et al. 2001; Izotov et al. 2001a) and, more impressively, by Vílchez \& Iglesias-Páramo (1998) who were the first to present a 2D spectroscopic study of the chemical abundance patterns of the warm interstellar medium (ISM) in I Zw 18.

Much less attention has been attracted by the fainter detached C component of I Zw 18 (hereafter I Zw 18 C), located $\sim 22^{\prime \prime}$ northwest of region NW. Dufour et al. (1996a), Petrosian et al. (1997), Izotov \& Thuan (1998a), van Zee et al. (1998) and Izotov et al. (2001a) have shown it to have the same recession velocity as the main body, thus establishing its physical association to I Zw 18. This was also shown through interferometric $21 \mathrm{~cm}$ studies (van Zee et al. 1998; see also Viallefond et al. 1987) which revealed that I Zw 18 and I Zw 18 C are immersed within a large common $\mathrm{HI}$ complex with a projected size of $60^{\prime \prime} \times 45^{\prime \prime}$ connecting with a $\gtrsim 1^{\prime}$ southern tail with no optical counterpart. The SF activity in I Zw $18 \mathrm{C}$ is known to be weak with its $E W(\mathrm{H} \alpha)$ not exceeding $\sim 60 \AA$ along its major axis (Izotov et al. 2001a, see also van Zee et al. 1998). Despite deep Keck II spectroscopy, Izotov et al. (2001a) failed to detect oxygen lines, so its oxygen abundance is not known.

Traditionally, the distance to I $\mathrm{Zw} 18$ has been taken to be $10 \mathrm{Mpc}$, assuming a pure Hubble flow recessional velocity. 
However, Izotov et al. (1999a), based on an HST colormagnitude diagram (CMD) study, have argued that the distance to I Zw 18 has to be at least $15 \mathrm{Mpc}$, and most likely $\sim 20 \mathrm{Mpc}$, in order for its brightest stars being massive enough to account for the ionizing flux observed. This upper distance value has recently received independent support by Fiorentino et al. (2010). These authors identify three long-period Cepheid candidates in I Zw 18, which, if interpreted as classical Cepheids, imply a distance of $19.0_{-2.5}^{+2.8} \mathrm{Mpc}$ by the Wesenheit relation. In the following, we adopt a distance $D=19 \mathrm{Mpc}$ to both I Zw 18 and I Zw $18 \mathrm{C}$ throughout and convert distance-dependent quantities from the literature accordingly. It should be noted, however, that the assumed distance has practically no influence on the main conclusions from this study.

The wealth of dedicated studies of I Zw 18 highlight the importance placed on this XBCD as a precious nearby laboratory for exploring collective star formation and the associated feedback process under metallicity conditions approaching those in distant protogalactic systems. Some examples include (i) the consideration of I Zw 18 as reference object for many dwarf galaxy chemical evolution models (Matteucci \& Tosi 1985; Roy \& Kunth 1995; Mas-Hesse \& Kunth 1999; Legrand 2000; Recchi et al. 2004, among others); (ii) the great deal of effort put in determining its chemical abundance patterns in its neutral ISM (Kunth et al. 1994; Aloisi et al. 2003; Lecavelier des Etangs et al. 2004); (iii) the study of its dust and molecular gas content (e.g. Cannon et al. 2002; Leroy et al. 2007); (iv) the thorough exploration of the excitation mechanisms of its brightest HII region (Stasińska \& Schaerer 1999; Péquignot 2008); and (v) the deep spectroscopic studies that led to the discovery of Wolf-Rayet stellar features in it (Izotov et al. 1997b; Legrand et al. 1997).

But arguably, the most longstanding debate associated with I Zw 18 ever since its discovery concerns its evolutionary status. In this regard, various interpretations have been put forward, ranging from I Zw 18 being a bona fide young galaxy, currently forming its first stellar generation (Sargent \& Searle 1970, see also Izotov \& Thuan 1999), through the diametrically opposite picture of an ancient "slowly cooking" dwarf galaxy that is forming stars continuously over the Hubble time (Legrand 2000; Legrand et al. 2001). Notwithstanding an impressive amount of high-quality multiwavelength data and numerous dedicated analyses of considerable effort and sophistication, the convergence towards a consensual view on the evolutionary status of I Zw 18 has been slow.

CMD analyses, based on HST data, have primarily been focusing on the question of whether I Zw 18 contains a sizeable population of evolved red giant branch (RGB) stars, similar to typical $(12+\log (\mathrm{O} / \mathrm{H}) \gtrsim 8$, see e.g. Kunth \& Östlin 2000) BCDs. In the latter, an extended envelope of resolved RGB stars around the SF component (e.g. Tosi et al. 2001; Crone et al. 2002) nicely echoes the since-long observationally established fact of an evolved underlying host galaxy in these systems (e.g. Loose \& Thuan 1986; Papaderos et al. 1996a; Cairós et al. 2001; Bergvall \& Östlin 2002; Noeske et al. 2003; Gil de Paz $\&$ Madore 2005). Initial CMD studies suggested an age for the main body between several 10 Myr (Hunter \& Thronson 1995; Dufour et al. 1996b) and $\sim 1$ Gyr (Aloisi et al. 1999). Östlin (2000) argues from an HST NICMOS near infrared (NIR) study that a fit to the $J$ vs. $J-H$ CMD of I Zw 18 is best achieved for a stellar population of age $\sim 5$ Gyr. The subsequent identification of five carbon star candidates with an estimated age 0.5-1 Gyr by Östlin \& Mouhcine (2005) is in accord with that conclusion, even though the number of evolved star candidates in all above studies (about a dozen altogether) was recognized to be surprisingly small compared to typical BCDs of equal luminosity. A significant step forward has been possible through the advent of HST ACS, allowing point source photometry to be extended to magnitudes as faint as 29 mag in the $V$ and $I$ bands and the question of the presence of RGB stars in I Zw 18 to be revisited (Izotov \& Thuan 2004, hereafter IT04). IT04 found, in addition to numerous blue main sequence and blue and red supergiants with an age $\lesssim 100 \mathrm{Myr}$, an older population of asymptotic giant branch stars with an age between 0.1 and 0.5 Gyr. This study, in which no RGB stars were detected, has been the first to also explore the spatial distribution of stars of different ages in I Zw 18. The upper age limit of $0.5 \mathrm{Gyr}$ for the oldest stars in I Zw 18 (IT04) was subsequently relaxed from a reanalysis of the same data by Yakobchuk \& Izotov (2006) that reveals an untypically small number of RGB candidates. Various other efforts have been made to improve on the CMD analysis of IT0 4 by pushing point source photometry to fainter levels by 1-2 mag (Momany et al. 2005; Tosi et al. 2007; Tikhonov 2007). The faintest ( $>29 \mathrm{mag}$ ) point sources in those CMDs almost uniformly cover the color range between $<-1$ mag and $>2$ mag. It is worth pointing out that, although divergent in their conclusions regarding stellar age, all CMD analyses for I Zw 18 consistently indicate a conspicuous absence of an extended stellar LSB envelope surrounding regions NW\&SE, in sharp contrast to any previously studied BCD.

As for I Zw $18 \mathrm{C}$, CMD analyses yield an upper age between a few ten and hundred Myr (Dufour et al. 1996b; Aloisi et al. 1999). Recently, Jamet et al. (2010) has employed a probabilistic CMD modeling technique and reports an upper age of $\sim 125 \mathrm{Myr}$, without, however, strictly ruling out the presence of older stars. An age of the same order was previously inferred for I Zw $18 \mathrm{C}$ from a combined CMD and evolutionary spectral synthesis study by Izotov et al. (2001a).

From the viewpoint of surface photometry, diametrically different conclusions on the photometric structure and evolutionary status of I Zw 18 were drawn by Kunth \& Östlin (2000, hereafter KÖ00) and Papaderos et al. (2002, hereafter P02). Nevertheless, these two studies were the first to demonstrate on the basis of surface photometry that I Zw 18 is not presently forming its first stellar generation but contains a substantial unresolved stellar background of intermediate age.

As a matter of fact, much of the disparity between these studies has stemmed from the differing importance they ascribed to the presence and photometric impact of ne. KÖO0 concluded that SF activity in I Zw 18 is hosted by an old, extended stellar disk that dominates the stellar mass, just like in typical BCDs. Their rationale has mainly been based on their finding that I Zw 18 shows an exponential intensity decrease and reddish ( $B-R \approx 0.6 \mathrm{mag}$ ) colors in its LSB envelope $\left(9^{\prime \prime} \gtrsim R^{\star} \lesssim 20^{\prime \prime}\right)$. On the assumption that stellar emission dominates throughout, this color translates via a continuous star formation model to an age of $\gtrsim 5$ Gyr. The central surface brightness $\mu_{0}$ and exponential scale length $\alpha$ of the disk, read off the $B$ band surface brightness profile (SBP) of KÖ00, imply that the old stellar host contains $\sim 1 / 2$ of the emission and the bulk of the stellar mass in I Zw 18. The stellar disk interpretation for I Zw 18 qualitatively agrees with the evolutionary scenario by Legrand et al. (2001, see also Legrand 2000). These authors argue that the low and uniform gas-phase metallicity of I Zw 18 can be reproduced through continuous low-level star formation throughout the main HI complex of I Zw $18\left(45^{\prime \prime} \times 60^{\prime \prime}\right)$ over the past $\sim 14$ Gyr. This process would produce an extended stellar disk of extremely low surface brightness $\left(\bar{\mu} \simeq 28 \mathrm{Bmag} / \square^{\prime \prime}\right)$. 
P02 (see also Papaderos et al. 2001), on the other hand, called the conclusions by KÖO0 into question by invoking various lines of observational evidence. First, they have empirically shown that an exponential outer intensity drop off is a generic property of the nebular halo of starbursting dwarf galaxies. Consequently, the exponentiality of the LSB envelope of I Zw 18 is not per se a compelling argument for it being a stellar disk. This also applies to its reddish colors, which can naturally be accounted for by photoionized gas of subsolar metallicity (see e.g. Krüger et al. 1995).

Second, and in a more straightforward approach, P02 used HST WFPC2 narrow band images to bidimensionally subtract the [OIII] and $\mathrm{H} \alpha$ line emission from broad band HST data in order to isolate and study the residual stellar emission in I Zw 18. This correction led to the virtual removal of the filamentary LSB envelope, proving its gaseous nature. Specifically, P02 have shown that ne already dominates the line-of-sight intensity at a photometric radius $R^{\star} \gtrsim 6^{\prime \prime}$ and contributes between $30 \%$ and $50 \%$ of the $R$ band luminosity of I Zw 18 .

After decontamination from nebular line emission (though still affected by nebular continuum emission), broad band images were then used to study the photometric structure and color distribution of the stellar component of I Zw 18. SBPs computed from them have revealed a very compact host that, in sharp contrast to typical BCDs, shows practically no radial color gradients and overall very blue $(\lessgtr 0.1 \mathrm{mag})$ colors down to a limiting surface brightness $\mu \sim 26 \mathrm{mag} / \square^{\prime \prime}$.

These exceptional properties were interpreted as evidence of youth: young stars in I Zw 18 did not have had enough time to migrate significantly far from their initial locus and gradually form the extended stellar host that is typical of evolved BCDs. This, and the blue optical and NIR colors of the southeastern tip of I Zw 18 (region $\omega$ in the notation of P02, cf. Fig. 1) where ne is weak led P02 to conclude that most of the stellar mass in I Zw 18 has formed within the past $0.5 \mathrm{Gyr}$. Hence, the picture put forward by $\mathrm{P} 02$ is that I Zw 18 is a cosmologically young object that presently undergoes its dominant formation phase and contains a small, if any, mass fraction of stars older than $\sim 1 \mathrm{Gyr}$. Further support for this conclusion came from a subsequent NIR study of I Zw 18 by Hunt et al. (2003).

As for I Zw $18 \mathrm{C}$, the nearly constant blue colors $(\approx 0 \mathrm{mag})$ determined within its Holmberg radius, lent further support to the youth interpretation that was previously advocated by Izotov et al. (2001a).

However, important aspects of the I Zw 18 system could not be conclusively addressed in previous photometric studies. For example, whereas SBPs for I Zw $18 \mathrm{C}$ by $\mathrm{P} 02$ reach a surface brightness level $\mu \sim 28 \mathrm{Bmag} / \square^{\prime \prime}$, their large photometric uncertainties already below $\approx 26 \mathrm{mag} / \square^{\prime \prime}$ have practically prevented an assessment of the question of whether a redder underlying stellar population dominates in the extremely faint periphery of the galaxy. Clearly, this issue is central to understanding the evolutionary status of I $\mathrm{Zw} 18 \mathrm{C}$. One may argue that, since the evolved stellar host generally dominates for $\mu \gtrsim 24.5 \mathrm{~B} \mathrm{mag} / \square^{\prime \prime}$ (Papaderos et al. 1996a, hereafter P96a), it would have been detected in I Zw $18 \mathrm{C}$, if present. This is a circular argument, however, given that empirical relations established for typical BCDs should not be taken for granted for young dwarf galaxy candidates. Similarly, due to the shallowness of previous surface photometry no definite conclusions could be drawn regarding the ultra-LSB disk predicted by Legrand (2000).

As for the main body of I $\mathrm{Zw} \mathrm{18,} \mathrm{previous} \mathrm{studies} \mathrm{did} \mathrm{not}$ have the sensitivity to pin down the maximal extent, morphology, and color pattern of the LSB envelope, adding potentially important constraints to chemodynamical and spectrophotometric models for I $\mathrm{Zw} 18$. From such considerations, extremely deep surface photometry appears crucial to further advancing our understanding of the photometric structure and evolutionary status of I Zw 18 and I Zw 18 C. This is particularly true for deep $I$ band surface photometry, which is entirely lacking both for I Zw 18 and I Zw $18 \mathrm{C}$. The $I$ band HST WFPC2 images included the main body of I Zw 18 only and were not deep enough for a study of its LSB envelope. This data set was therefore not used in the surface photometry analysis by $\mathrm{P} 02$.

This study is motivated by the availability of an unprecedently deep set of archival HST ACS $V, R$, and $I$ broad band data that has accumulated over the past few years. $I$ band photometry is an important asset in this respect, not only due to its higher sensitivity to a putative old stellar background but also because it offers, in combination with $V$ and $R$ data, a sensitive tracer of ne both in the center and the LSB envelope of I Zw 18. This is because the $I$ band is affected by nebular continuum emission alone, whereas the $V$ and $R$ transmission curves additionally include, respectively, the strong [OII] $\lambda \lambda 44959,5007$ and $\mathrm{H} \alpha$ emission lines. As a result, regions with strongly enhanced ne can be identified readily by their extremely blue $(-0.5 \ldots-1.4) V-I$ and $R-I$ colors (see e.g. Papaderos et al. 1998, P02 and references therein for a discussion and examples among XBCDs).

This paper is organized as follows. In Sect. 2 we discuss the data processing and SBP derivation technique used and in Sect. 3 the structural and morphological properties of $\mathrm{I} \mathrm{Zw} 18 \mathrm{C}$ and I $\mathrm{Zw} 18$. Section 4 concentrates on the evolutionary status and the formation process of I Zw $18 \mathrm{C}$ (Sect. 4.1) when considering the effect that the diffusion of young stars would have on the observed colors (Sect. 4.1.1). The evolutionary status of I Zw 18 and the hypothesis of an ultra-LSB underlying stellar disk are discussed on the basis of the present photometric analysis in Sects. 4.2 and 4.2.1, respectively. In Sect. 4.3 we use I Zw 18 as template to briefly explore the biases that extended ne may introduce in photometric studies of morphologically analogous SF galaxies at higher redshift $(z)$. The main results from this study are summarized in Sect. 5 .

\section{Data processing}

This study is based on the entire set of archival HST ACS broad band images for I Zw 18 that has been acquired through the HST programs 9400 (PI: Thuan) and 10586 (PI: Aloisi). It comprises 38,65 and 81 images in the filters $F 555 W(V), F 606 W$ (broad $V R$, referred to in the following as $R$ ) and $F 814 W(I)$, summing up to on-source exposures of 87,55 , and $101 \mathrm{ks}$, respectively. This is the deepest imaging data set currently available for I Zw 18, with an integration time in $R$ and $I$ equaling about one third of the time spent on the HST ACS Ultra Deep Field (Beckwith et al. 2006) in the filters $F 606 W$ and $F 775 W$. The data processing was carried out using IRAF ${ }^{1}$ and ESO MIDAS ${ }^{2}$. Photometric quantities refer to the Vega system.

Since the main goal of this study is deep surface photometry, its most critical aspect is the removal of diffuse and compact background sources (diffuse extragalactic background, zodiacal light, foreground stars, and background galaxies, respectively).

1 IRAF is the Image Reduction and Analysis Facility distributed by the National Optical Astronomy Observatory, which is operated by the Association of Universities for Research in Astronomy (AURA) under cooperative agreement with the National Science Foundation (NSF).

2 Munich Image Data Analysis System, provided by the European Southern Observatory (ESO). 


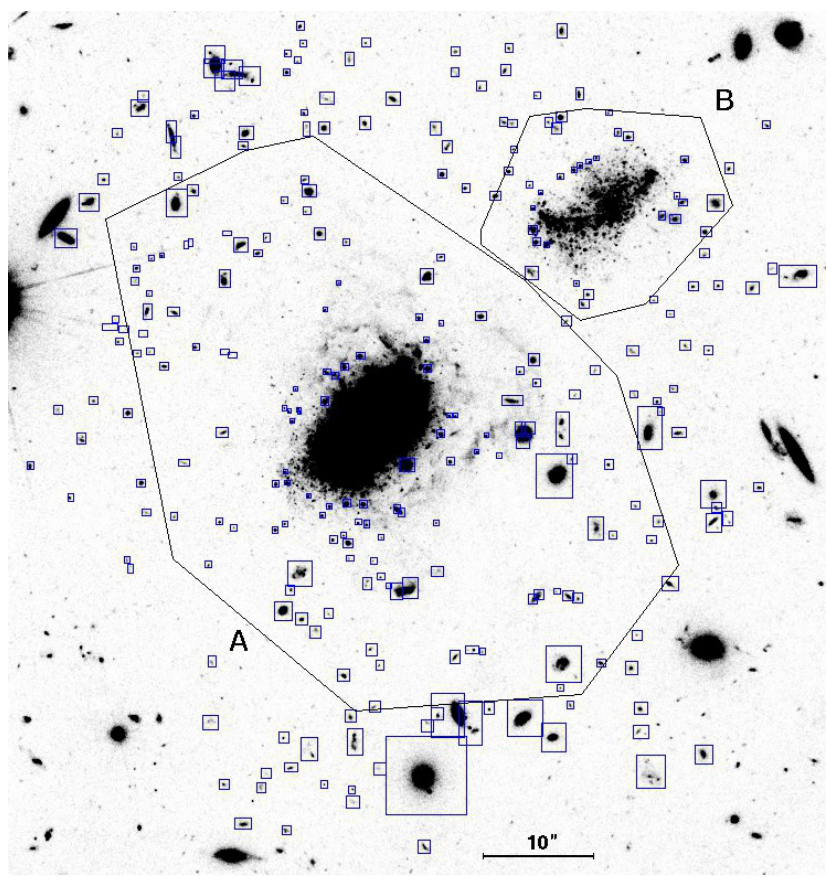

Fig. 2. Combined $I$ band exposure of I Zw 18 and I Zw $18 \mathrm{C}$ with pointand compact sources in the vicinity of the galaxies (polygonal regions $\mathrm{A}$ and B) marked with rectangles. North is up and east to the left.

After subtracting the diffuse background, we therefore checked to what extent compact or point sources (cps) within the extended LSB envelope of I Zw 18 can affect SBPs and color profiles. For example, for an extended source of constant surface brightness $\mu=29 \mathrm{mag} / \square^{\prime \prime}$, the total apparent magnitude $m$ of a circular annulus with $19^{\prime \prime} \leq R^{\star} \leq 20^{\prime \prime}$ (roughly the radius of I Zw 18) is $23.8 \mathrm{mag}$. At this intensity level, already a single faint ( $m=25 \mathrm{mag}$ ) background cps can introduce an error of $0.3 \mathrm{mag}$ in surface photometry. We adopted the following procedure: after image alignment and correction for cosmics, we used the combined images in the three filters to compile a catalog of cps in the relevant portion of the field of view. In doing this, we disregarded cps in I Zw 18 (roughly the area subtended by the $25 R^{\prime} \mathrm{mag} / \square^{\prime \prime}$ isophote in Fig. 8) and in I Zw $18 \mathrm{C}$, as well as compact clumps of ne, identified by their blue $V-I$ and $R-I$ color. All cps in each frame were in turn replaced by the mean intensity in the adjacent area. Special care was given to the removal of two background galaxies close to the western supershell of I Zw 18. This was done by subtracting a 2D model, computed with the Bender \& Möllenhoff (1987) algorithm. In total, $\sim 140$ cps were removed in the field of interest around I Zw 18 and I Zw $18 \mathrm{C}$ (polygonal regions labeled A and B in Fig. 2). Their integral $I$ magnitude of $19.17 \mathrm{mag}$ and $21.3 \mathrm{mag}$ within the regions considered $\left(\sim 1600 \square\right.$ " and $\sim 200 \square^{\prime \prime}$ for A and B, respectively) corresponds to a mean surface brightness of $\sim 27$ I $\mathrm{mag} / \square^{\prime \prime}$. This value is consistent at the $1.5 \sigma$ level with the value of 26.7 I mag/ $\square^{\prime \prime}$ inferred by Zackrisson et al. (2009) for the resolved extragalactic background light emission. The isophotal radius of the stellar component of $\mathrm{I} \mathrm{Zw} 18$ and $\mathrm{I} \mathrm{Zw} 18 \mathrm{C}$ at $\mu=26 \mathrm{Bmag} / \square^{\prime \prime}$ was determined by P02 to be $8 . .8$ and $5^{\prime \prime} .5$, respectively. From their combined isophotal area of $\sim 340 \square^{\prime \prime}$ and the above derived cps surface density of $\sim 0.08 \square^{\prime \prime-1}$, we expect about 27 background cps in I Zw 18 and I Zw $18 \mathrm{C}$ altogether. The SBPs computed prior to and after removal of cps were found in all bands to be consistent within $1 \sigma$ uncertainties, ensuring that cps contamination does not affect our conclusions in Sect. 3.
SBPs were computed with the code iv (P02) (also referred to as Lazy by Noeske et al. 2006) that was specifically developed for the study of irregular galaxies. This code permits a simultaneous processing of co-aligned images of a galaxy in several bands and does not require a choice of a galaxy center. It also does not implicitly assume that the galaxy can be approximated by the superposition of axis-symmetric luminosity components. One of its key features is the computation of photon statistics within automatically generated irregular annuli that are adjusted to the galaxy morphology for each surface brightness interval $\mu \pm \Delta \mu$. This distinguishes code iv from other surface photometry packages that generally employ ellipse-fitting to isophotes or photon statistics within elliptical annuli (e.g. meth. i of P96a, task ELLIPSE in IRAF, FIT/ELL3 in MIDAS), or approximate a galaxy by a single or several $2 \mathrm{D}$ axis-symmetric components (e.g. GIM2D, Simard 1998, and GALFIT, Peng et al. 2002).

\section{Results}

\subsection{The photometric structure of I Zw $18 \mathrm{C}$}

In this section we investigate the photometric structure of I Zw $18 \mathrm{C}$, based on the combined HST ACS data in the filters $V$, $R$, and $I$. These allow extending previous HST WFPC2 surface photometry (P02) by $\gtrsim 1 \mathrm{mag}$, with significantly reduced uncertainties below $\mu \sim 26 \mathrm{mag} / \square^{\prime \prime}$. In agreement with previous work, the SBPs of I Zw $18 \mathrm{C}$ in all bands (Fig. 3a) were found to be nearly indistinguishable from one another, implying nearly constant radial colors. All SBPs display a narrow $\left(R^{\star} \leq 1^{\prime \prime}\right)$ central excess and an outer $\left(3^{\prime \prime} \lesssim R^{\star} \lesssim 9^{\prime \prime}\right)$ roughly exponential drop-off that mainly reflects the luminosity output from the host galaxy. A salient feature at intermediate radii $\left(1^{\prime \prime} \leq R^{\star} \lesssim 3^{\prime \prime}\right)$ is a shallower intensity increase than what the inward extrapolation of the outer profile slope to $R^{\star}=0^{\prime \prime}$ predicts. An adequate fit to such SBPs therefore requires a modified exponential model that involves an extended $\left(R^{\star} \sim 3^{\prime \prime}\right)$ central core of nearly constant surface brightness. One such fitting function (hereafter modexp), used by P96a to fit the host galaxy of BCDs (see Noeske et al. 2003, for applications on near infrared (NIR) studies and a comparison with the Sérsic law) takes the form

$I\left(R^{*}\right)=I_{\exp }\left[1-\epsilon_{1} \exp \left(-P_{3}\left(R^{*}\right)\right)\right]$

where $P_{3}\left(R^{*}\right)$ is defined as

$P_{3}\left(R^{*}\right)=\left(\frac{R^{*}}{\epsilon_{2} \alpha}\right)^{3}+\left(\frac{R^{*}}{\alpha} \frac{1-\epsilon_{1}}{\epsilon_{1}}\right)$.

In addition to the central intensity $I_{0}$ and scale length $\alpha$ of a pure exponential profile $I_{\exp }=I_{0} \exp \left(R^{*} / \alpha\right)$, Eqs. (1) and (2) involve two other parameters: the central intensity depression $\epsilon_{1}=\Delta I / I_{0}$ relative to an exponential model and the core radius $R_{\mathrm{c}}=\epsilon_{2} \alpha$. The best-fitting modexp model to the $V$ band SBP for $\epsilon_{1,2}=(2.4,0.8)$ (cf. P02) is shown in Fig. 3a. It yields in all bands an extrapolated central surface brightness $\mu_{\mathrm{E}, 0}=21.7 \pm 0.2$, a true central surface brightness $\mu_{0}=\mu_{\mathrm{E}, 0}-$ $2.5 \log \left(1-\epsilon_{2}\right)=23.45 \mathrm{mag} / \square^{\prime \prime}$ and an $\alpha$ in the narrow range between 108 and 117 pc. The absolute $V$ magnitude determined from the modexp fit $(-11.67 \mathrm{mag})$ corresponds to $\sim 80 \%$ of the total luminosity of I Zw $18 \mathrm{C}$.

A direct determination of the intensity profile of the host galaxy of BCDs for radii $R^{\star} \leq R_{\mathrm{c}}$ is generally prevented by the luminous young stellar component that typically dominates out to $R_{\mathrm{SF}} \approx 2 \alpha$ (Papaderos et al. 1996b, hereafter P96b). Since young stellar clusters (SCs) can hardly be sufficiently resolved 

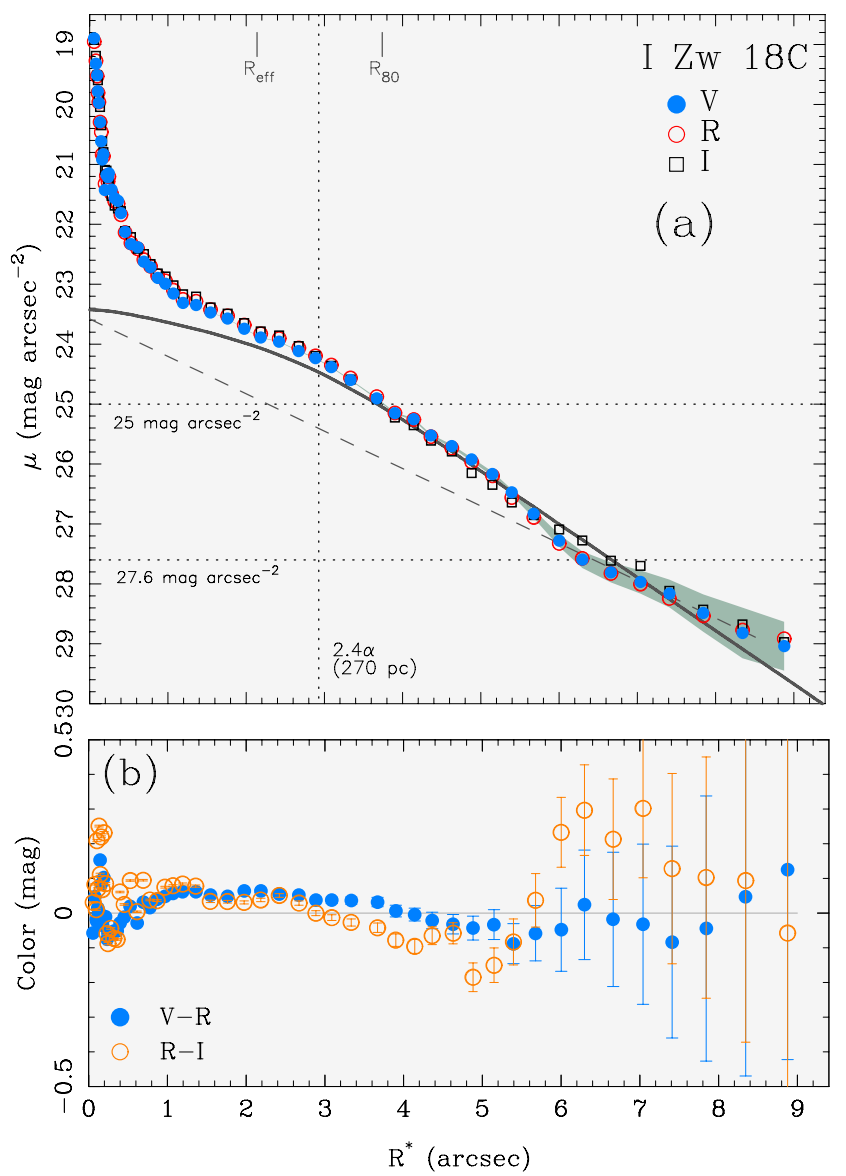
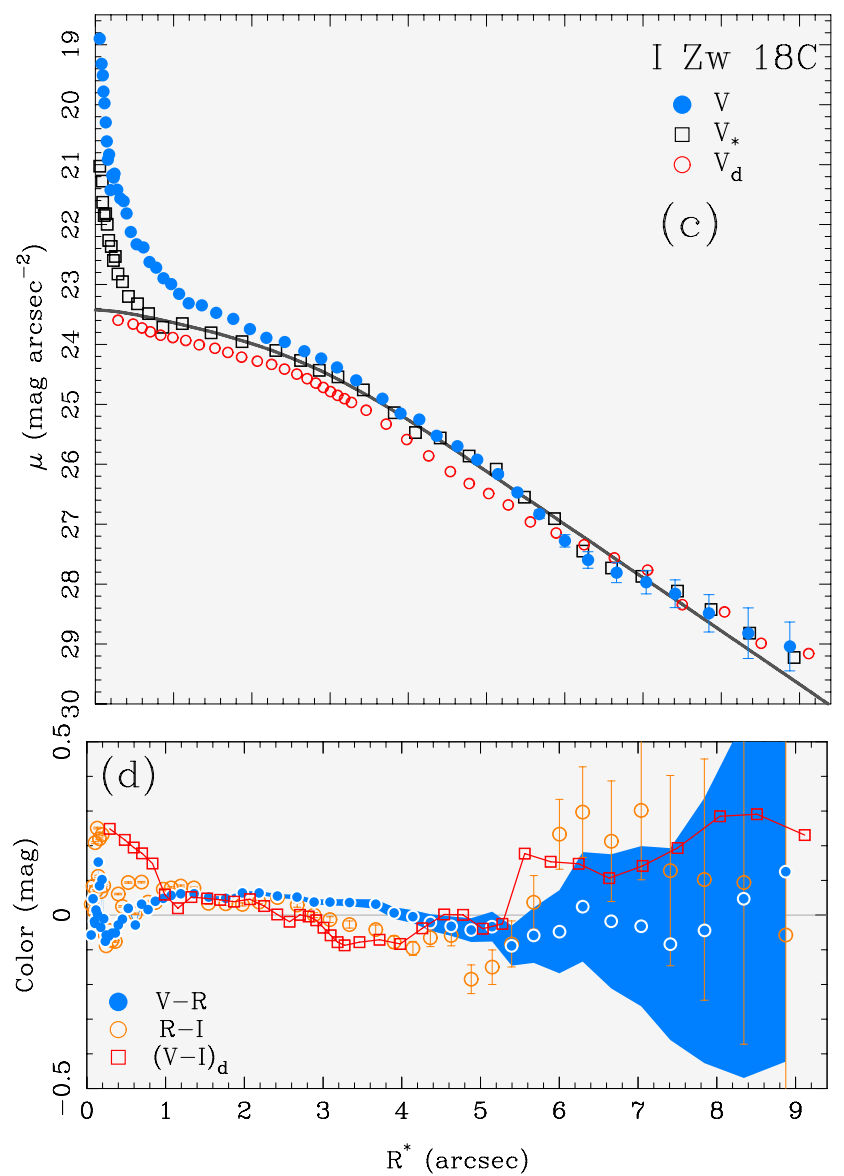

Fig. 3. a) Surface brightness profiles (SBPs) of I Zw $18 \mathrm{C}$ in $V(F 555 W), R(F 606 W)$ and $I(F 814 W)$. The thick gray curve shows a fit to the $V$ SBP for $R^{\star} \geq 33^{\prime \prime} 8$ with the modified exponential fitting function Eq. (1) (modexp) for a core radius $R_{\mathrm{c}}=2.4 \cdot \alpha$ (dotted vertical line). The effective radius $R_{\text {eff }}$ and the radius $R_{80}$ enclosing $80 \%$ of the total $V$ emission are indicated. The dashed line shows a linear fit to the outermost exponential part of the $V$ SBP for $R^{\star} \geq 6^{\prime \prime}$, i.e. at the extremely faint $\left(\mu \geq 27.6 \mathrm{mag} / \square^{\prime \prime}\right)$ outskirts of the galaxy. The shadowed area corresponds to the $1 \sigma$ uncertainties of the $V$ SBP. b) Radial $V-R$ and $R-I$ color profiles of I Zw $18 \mathrm{C}$, derived from the SBPs in the upper panel. c) Comparison of the best-fitting modexp model in panel a) (thick gray curve) with the $V$ SBPs of I Zw $18 \mathrm{C}$ after partial removal of the brightest point sources ( $V_{\star}$; squares). Open circles show the SBP of the unresolved stellar emission $\left(V_{\mathrm{d}}\right)$, computed after complete removal of compact $(\leq 0$.'5) sources with an unsharp masking technique. d) Comparison of the color profiles in panel b) (open and filled circles) with the $(V-I)_{\mathrm{d}}$ color profile (squares) of the unresolved stellar emission. The shadowed area depicts the $1 \sigma$ uncertainties of the $V-R$ profile.

and subtracted out, even at the angular resolution of the HST, the chosen parameter set $\epsilon_{1,2}$ has to rely on plausibility arguments (see discussion in P96a and Noeske et al. 2003) and is only to be considered approximative.

In the case of I $\mathrm{Zw} 18 \mathrm{C}$, however, due to the comparatively low surface density of young SCs, one stands a better chance of directly constraining the central form of the host's SBP. For this, we first subtracted out the brightest $\sim 200$ point sources from the galaxy using DAOPHOT (Stetson 1979) and subsequently recomputed the $V$ SBP from the residual emission. Figure $3 \mathrm{c}$ shows that this SBP closely matches the best-fitting modexp model for intermediate to large $R^{\star}$. This agreement suggests that the adopted $\epsilon_{1,2}$ parameters yield a reasonable first-order approximation to the unresolved emission of the host. SBP integration indicates that roughly $30 \%$ of I Zw 18 C's emission within $R_{80}$ (Fig. 3) is due to point sources. This is rather a lower limit for the luminosity fraction of cps given that with the adopted procedure SCs could not be fully deblended and subtracted out. This is apparent from the still strong ( $2 \mathrm{mag})$ central peak of the $V_{\star}$ SBP that is mainly due to incomplete removal of the central SC complex $\mathrm{C}$ and surrounding bright SCs (cf. Fig. 4a).

In an effort to better constrain the SBP of the unresolved stellar component of I $\mathrm{Zw} 18 \mathrm{C}$, we subsequently applied a flux-conserving unsharp masking technique (Papaderos et al. 1998, hereafter P98) to filter out all higher-surface brightness (HSB) clumpy features with $\leq 0$ '. 5 and isolate the diffuse $V$ emission. The frames holding the compact $\left(V_{\mathrm{c}}, R_{\mathrm{c}}\right.$, and $\left.I_{\mathrm{c}}\right)$ and diffuse $\left(V_{\mathrm{d}}, R_{\mathrm{d}}\right.$, and $\left.I_{\mathrm{d}}\right)$ emission in each band are displayed in panels a and b of Fig. 4, respectively. The $V_{\mathrm{d}}$ SBP (Fig. 3c) is at intermediate radii $\left(1^{\prime \prime} \lesssim R^{\star} \lesssim 6^{\prime \prime}\right)$ fairly comparable to the $V_{\star} \mathrm{SBP}$, except for a nearly constant offset by $\approx 0.3 \mathrm{mag}$. Its corresponding absolute magnitude $(-11.3 \mathrm{mag})$ is by about $0.75 \mathrm{mag}$ fainter than the integral value for I Zw $18 \mathrm{C}(-12.05 \mathrm{mag})$ and can be regarded as characteristic for the unresolved stellar emission in the host galaxy.

All SBPs in Figs. 3a,c display at very faint levels $(\mu \gtrsim$ $\left.27.6 \mathrm{mag} / \square^{\prime \prime}\right)$ a shallower outer $\left(R^{\star} \gtrsim 6^{\prime \prime}\right)$ exponential slope (Fig. 3a). This feature is certainly not due to point spread function (PSF) convolution effects since the maximum extent of the ACS PSF at its lowest measured intensity (10 mag below its central value) is $\sim 1 "$ '5 (Jee et al. 2007). Nebular emission contamination from the main body can as well be excluded both on the basis of narrow-band imaging (e.g. Östlin et al. 1996, P02) and because it would make $V-I$ and $R-I$ color profiles bluer, in disagreement with their slightly redder values for $R^{\star} \gtrsim 6^{\prime \prime}$ (see below). This outermost SBP feature has evaded detection 
P. Papaderos and G. Östlin: I Zw 18 as morphological paradigm for rapidly assembling high-z galaxies
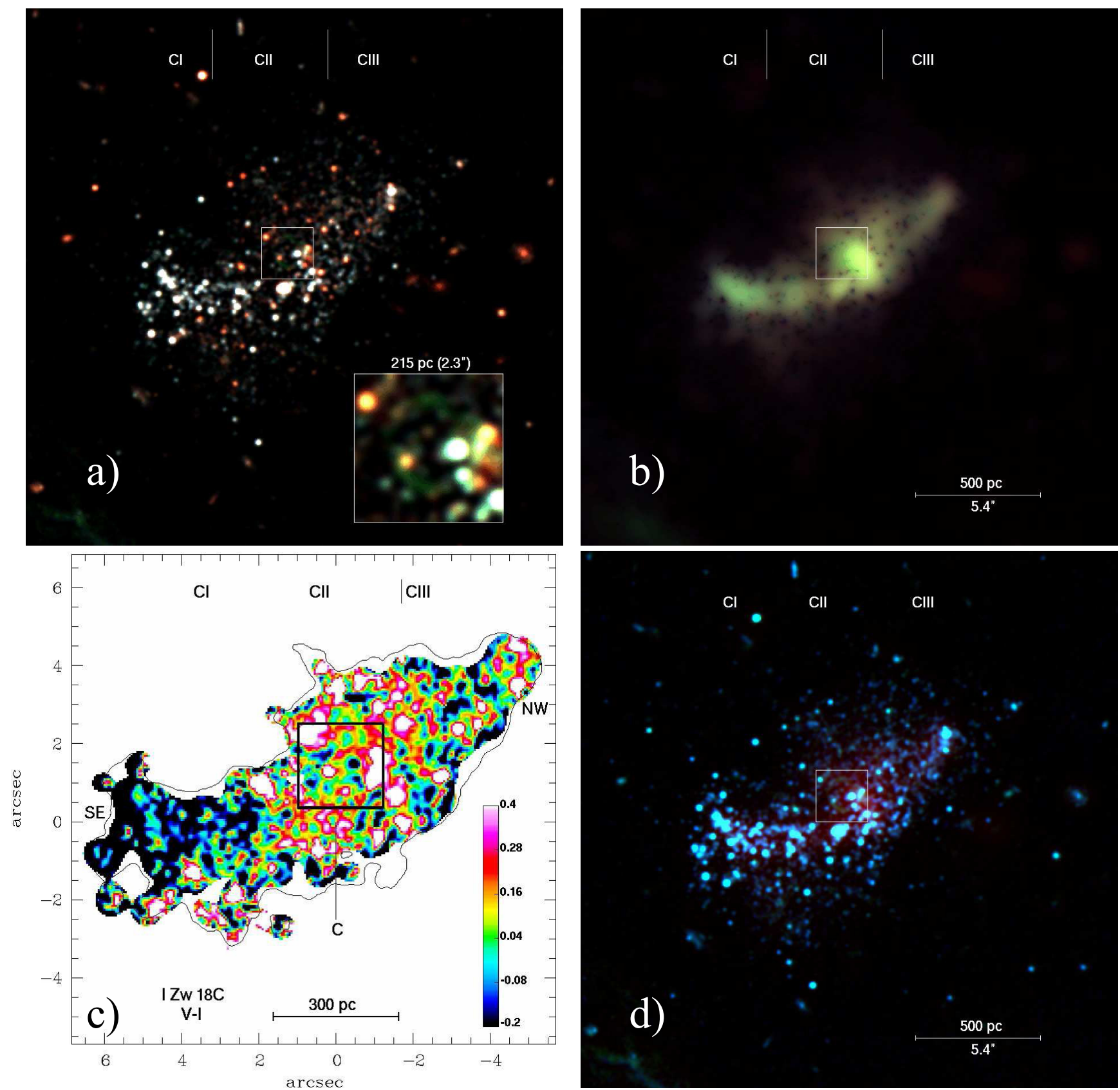

Fig. 4. a) Three-color composite of the images $R_{\mathrm{c}}, V_{\mathrm{c}}$ and $I_{\mathrm{c}}$ (red, green, and blue image channels, respectively), illustrating the spatial distribution of compact $(\leq 0,5)$ sources in I Zw $18 \mathrm{C}$. The regions $\mathrm{C}_{\mathrm{I}}, \mathrm{C}_{\text {II }}$, and $\mathrm{C}_{\text {III }}$ defined by IT04 are indicated. The blown up version of the central part of I Zw $18 \mathrm{C}$ (lower-right) shows the ionized gas shell in the vicinity of the bright stellar cluster C (cf. Dufour et al. 1996b). b) Three-color composite of $R_{\mathrm{d}}, V_{\mathrm{d}}$ and $I_{\mathrm{d}}$, of the unresolved stellar emission. The color coding is the same as in panel a). c) $V-I$ color map of I Zw $18 \mathrm{C}$, revealing very blue colors $(V-I<0)$ all over the southeastern third (region $\mathrm{C}_{\mathrm{I}}$ ) of the galaxy. The contour corresponds to $25 V$ mag/ $\square^{\prime \prime}$. d) Comparison of the spatial distribution of compact and point sources in $V_{\mathrm{c}}$ and $R_{\mathrm{c}}$ (blue and green channels, respectively) with the unresolved stellar background ( $I_{\mathrm{d}}$, red channel).

in previous surface photometry (P02) which, due to photometric uncertainties, was practically restricted to within the Holmberg radius. Because of its faintness (merely $3 \%$ of the total emission) and low surface brightness $\left(\sim 27.6-29 \mathrm{mag} / \square^{\prime \prime}\right)$, its reality cannot be established beyond doubt even with the present data. If an underlying stellar host of constant exponential slope to $R^{\star}=0^{\prime \prime}$, then its $\mu_{0}\left(\approx 23.6 \mathrm{mag} / \square^{\prime \prime}\right)$ and $\alpha(160 \mathrm{pc})$ would qualify it as an LSB dwarf with an $M_{V} \approx-11$ mag (equivalent to $\sim 38 \%$ of I Zw 18 C's emission).

We turn next to the color distribution in I Zw $18 \mathrm{C}$. Figure $3 \mathrm{~b}$ shows that, for $1^{\prime \prime} \lesssim R^{\star} \lesssim 6^{\prime \prime}$, both the $V-R$ and $R-I$ indexes are very blue and nearly constant $(\approx 0$ with a standard deviation about the mean of $0.05 \mathrm{mag}$ ). Since $R^{\star}=6^{\prime \prime}$ encompasses practically all the emission of I Zw $18 \mathrm{C}$, this color may be regarded as representative of the galaxy as a whole. In the outermost periphery of the galaxy, where the shallower outer exponential component appears, color profiles hold a hint of a slightly redder $R-I(0.16 \pm 0.09)$ without a notable change in $V-R$. The color profiles in their innermost $\left(R^{\star} \leq 1^{\prime \prime}\right)$ part are dominated by luminous cps in the surroundings of region C (cf. Fig. 1) and show a large scatter (up to $0.15 \mathrm{mag}$ ) around mean values of 0 mag for $V-R$ and 0.06 mag for $R-I$. 
To place meaningful constraints on the evolutionary status of I $\mathrm{Zw} 18 \mathrm{C}$, it is necessary to verify that these blue colors do not simply result from the luminosity-weighted average of bright blue SCs with a red underlying stellar background. The latter could readily escape detection, even when dominating the stellar mass (see e.g. P98). This concern is underscored by two atypical properties of I Zw 18 C: First, the galaxy exhibits a weak color gradient along its major axis (cf. Aloisi et al. 1999; Izotov et al. 2001a), with its northwestern tip being redder $(B-V=0.05 \mathrm{mag}$, $V-I=0.2 \mathrm{mag})$ than the southeastern one $(B-V=-0.07 \mathrm{mag}$ and $V-I=-0.2 \mathrm{mag}$ ). This is apparent from Fig. $4 \mathrm{c}$, from which a $V-I$ color as blue as -0.2 mag can be read off all over the southeastern third of the galaxy (region $\mathrm{C}_{\mathrm{I}}$ in the denomination by IT04). In contrast, the average $V-I$ color in the central (C II) and northwestern ( $\mathrm{C}$ III) part of the galaxy is redder ( $\geq 0 \mathrm{mag}$ ) with several local color maxima ( $\geq 0.3 \mathrm{mag}$ ) associated with cps. It is thus conceivable that the bluer southeastern and redder northwestern galaxy half counterbalance each other, thereby introducing an overall blue mean radial color. Another characteristic of $\mathrm{I} Z \mathrm{~W} 18 \mathrm{C}$ that could additionally conspire in diminishing radial color gradients is that the surface density of its cps tends to be spatially anti-correlated with the unresolved stellar background of the host. This is illustrated in Fig. 4d, where the diffuse emission of the latter $\left(I_{\mathrm{d}}\right.$ : red channel) is overlaid with the cps in the $V_{\mathrm{c}}$ and $R_{\mathrm{c}}$ images (blue and green channels, respectively). It can be seen that the diffuse component peaks at the northwestern half of I Zw $18 \mathrm{C}$ (C II and C III) in which it accounts for $\approx 50 \%$ of the $V$ line-of-sight intensity, whereas its contribution drops to $\$ 30 \%$ in the southeastern half of I Zw $18 \mathrm{C}$ (region $\mathrm{CI}_{\mathrm{I}}$ ) where most of the bright blue cps are located. Consequently, especially in region $\mathrm{C}_{\mathrm{I}}$, a hypothetical red stellar background could readily escape detection on radial color profiles.

From such considerations, and in order to infer the colors of the unresolved stellar component of I $\mathrm{Zw} 18 \mathrm{C}$ in an as unbiased manner as possible, we also computed color profiles based on $V_{\mathrm{d}}, R_{\mathrm{d}}$, and $I_{\mathrm{d}} \mathrm{SBPs}$. In all cases, we found good agreement with the results initially obtained from the total emission with the exception of a central $\left(R^{\star} \lesssim 1^{\prime \prime}\right)$ red peak with mean values of $(V-R)_{\mathrm{d}}=0.1 \pm 0.03$ and $(V-I)_{\mathrm{d}}=0.16 \pm 0.05$. This color excess might be attributed to a stellar age gradient or enhanced extinction in region $\mathrm{C}$ and surroundings. From spectral synthesis models, Izotov et al. (2001a) estimates an extinction coefficient $\mathrm{C}(\mathrm{H} \beta)=0.1-0.3$ for region $\mathrm{C}$, corresponding to $A_{V}=0.2-$ $0.65 \mathrm{mag}$.

As is apparent from panel $\mathrm{d}$ of Fig. 3, the $(V-I)_{\mathrm{d}}$ profile is fairly comparable to the $V-R$ and $R-I$ profiles (panel b), revealing a nearly constant color of $\approx 0 \pm 0.04$ mag within $1^{\prime \prime} \leq R^{\star} \leq 6^{\prime \prime}$ and a slightly redder value $(0.2 \pm 0.08 \mathrm{mag})$ in the extreme periphery of the galaxy. We are therefore led to conclude that the overall blue colors of I Zw $18 \mathrm{C}$ for $1^{\prime \prime} \lesssim R^{\star} \lesssim 6^{\prime \prime}$ are not dictated by bright young SCs spread over the body of the galaxy but characteristic of its unresolved stellar component.

\subsection{The photometric structure of I Zw 18}

The $V, R$, and $I$ SBPs of I Zw 18 (Fig. 5) compare well to those presented by KÖ00 and P02. They exhibit a central $\left(R^{\star} \lesssim 6^{\prime \prime}\right)$ HSB core and a nearly exponential LSB envelope extending out to $R^{\star} \sim 20^{\prime \prime}$. Profile fitting in the radius range $7^{\prime \prime} \leq R^{\star} \leq 15^{\prime \prime}$ yields an $\alpha=2$ '. $3(210 \mathrm{pc}$ ) and a luminosity fraction of $\approx 30 \%$ for the LSB component. At fainter levels ( $\left.\gtrsim 27.6-30 \mathrm{mag} / \square^{\prime \prime}\right)$ all SBPs exhibit a shallower exponential slope with $\alpha=4$ ". 1 (370 pc). This SBP feature is attributable to the faint filamentary emission in the extreme northwestern and southeastern

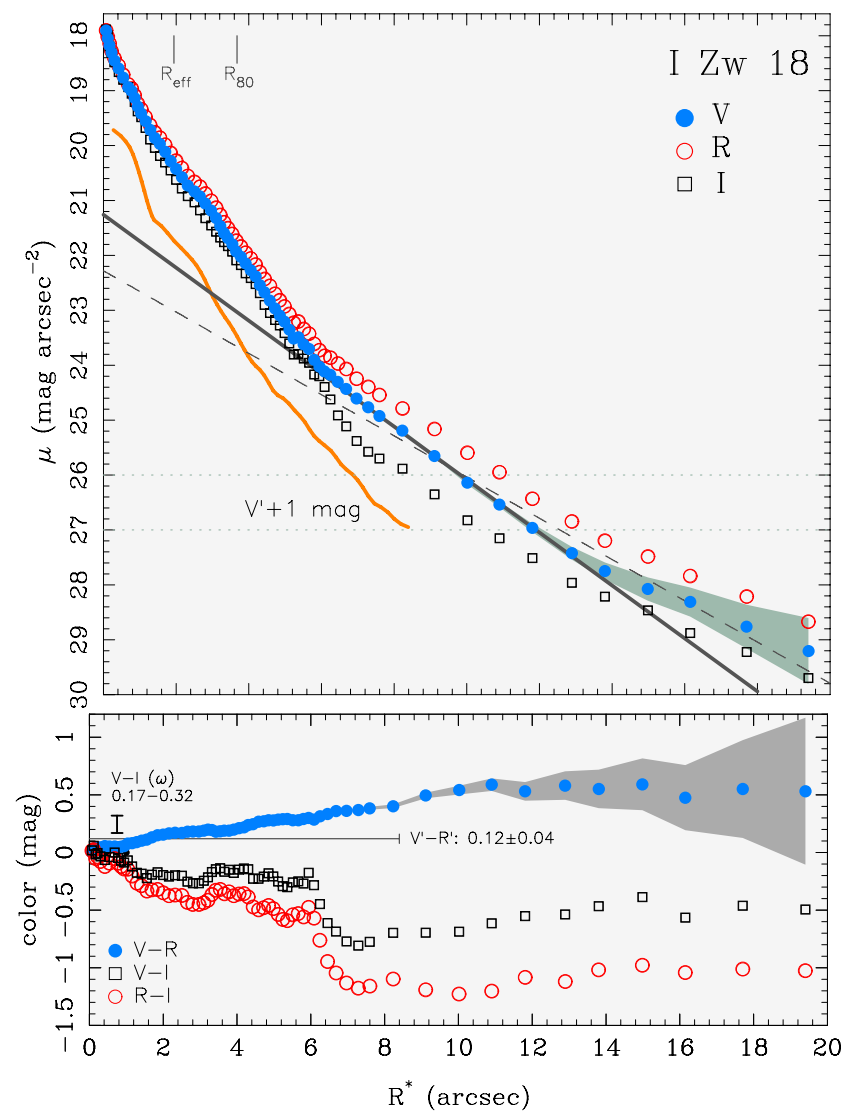

Fig. 5. (upper panel) $V, R$, and $I$ SBPs of I $\mathrm{Zw} 18$. The thick-solid and thin-dashed lines show linear fits to the $V$ SBP in the radius range $7^{\prime \prime} \leq R^{\star} \leq 15^{\prime \prime}$ and $R^{\star} \geq 7^{\prime \prime}$, respectively. The effective radius $R_{\text {eff }}$ and the radius $R_{80}$ enclosing $80 \%$ of the total $V$ emission are indicated. The $V$ SBP derived by $\mathrm{P} 02$ after subtraction of the $\mathrm{H} \beta$ and [OIII] $\lambda \lambda 4959$, 5007 emission lines (referred to as $V^{\prime}$ ), shifted by +1 mag for the sake of clarity, is included for comparison. (lower panel) $V-R$ (filled circles), $V-I$ (squares), and $R-I$ (open circles) color profiles, computed from the SBPs in the upper panel. The vertical bar indicates the $V-I$ color range inferred by P02 from ground-based and HST PC data for region $\omega$ at the southeastern tip of I Zw $18(V-I(\omega)=0.17 \ldots 0.32 \mathrm{mag})$. The mean $V-R$ color of I Zw 18's host after subtraction of nebular line emission $\left(V^{\prime}-R^{\prime}=0.12 \pm 0.04 \mathrm{mag} ; \mathrm{P} 02\right)$ is indicated by the horizontal line. The colors $V-I(\omega)$ and $V^{\prime}-R^{\prime}$ of the stellar host galaxy are by, respectively, $\geq 0.8$ mag redder and $\approx 0.4$ mag bluer than the colors of the exponential nebular envelope $\left(R^{\star} \geq 6^{\prime \prime}\right)$.

periphery of I Zw 18 (cf. Fig. 8) that has evaded detection on previous HST WFPC2 imagery and which accounts for no more than $1 \%$ of the total luminosity. A linear fit to the whole LSB envelope $\left(7^{\prime \prime} \leq R^{\star} \leq 20^{\prime \prime}\right)$ yields a mean $\alpha=22^{\prime \prime} 9 \pm 00^{\prime} \cdot 13(\sim 270 \mathrm{pc})$.

The radial color profiles of I Zw 18 (lower panel of Fig. 5) reflect the increasing contribution of ne to the observed line-ofsight intensity with increasing galactocentric radius, in agreement with previous evidence (P02). The $V-R$ color increases roughly linearly from $\lesssim 0$ at $R^{\star}=0^{\prime \prime}$ to $0.55 \mathrm{mag}$ at $R^{\star}=9^{\prime \prime}$ where it levels off to a nearly constant value. The corresponding, relatively strong color gradient of $\gamma_{+}=0.6 \mathrm{mag} \mathrm{kpc}^{-1}$ is fairly typical of evolved BCDs (P96b), suggesting, at first glance, that SF activity in I Zw 18 occurs within a more extended, old underlying host. This interpretation is, however, immediately challenged upon inspection of the extremely blue $V-I$ and $R-I$ profiles. The latter display already in their inner part $\left(2^{\prime \prime} \lesssim R^{\star} \leq 6^{\prime \prime}\right)$ mean values of -0.21 mag and -0.44 mag, respectively, both inconsistent with the red $V-R$ color, if the emission is assumed 

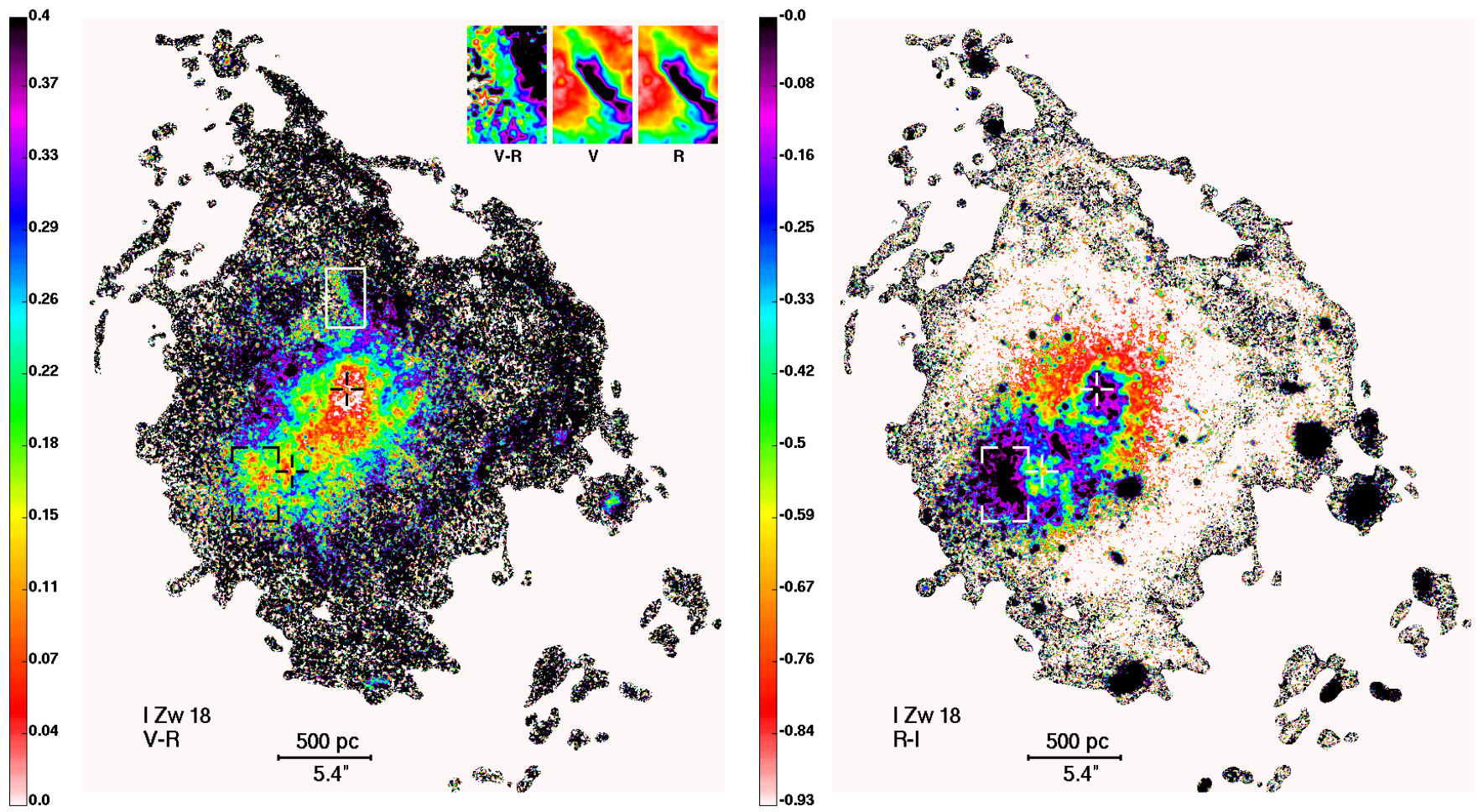

Fig. 6. $V-R$ (left panel) and $R-I$ (right panel) color map of I $\mathrm{Zw} 18$, displayed in the range between 0 and 0.4 mag and -0.9 and 0 mag, respectively. Crosses mark the SF regions NW and SE. The region termed $\omega$ by P02 (cf. Fig. 1) is indicated at the southeastern part of the image. The insets in the left panel show a magnified view of a shell $\approx 5^{\prime \prime}$ northward of region NW.

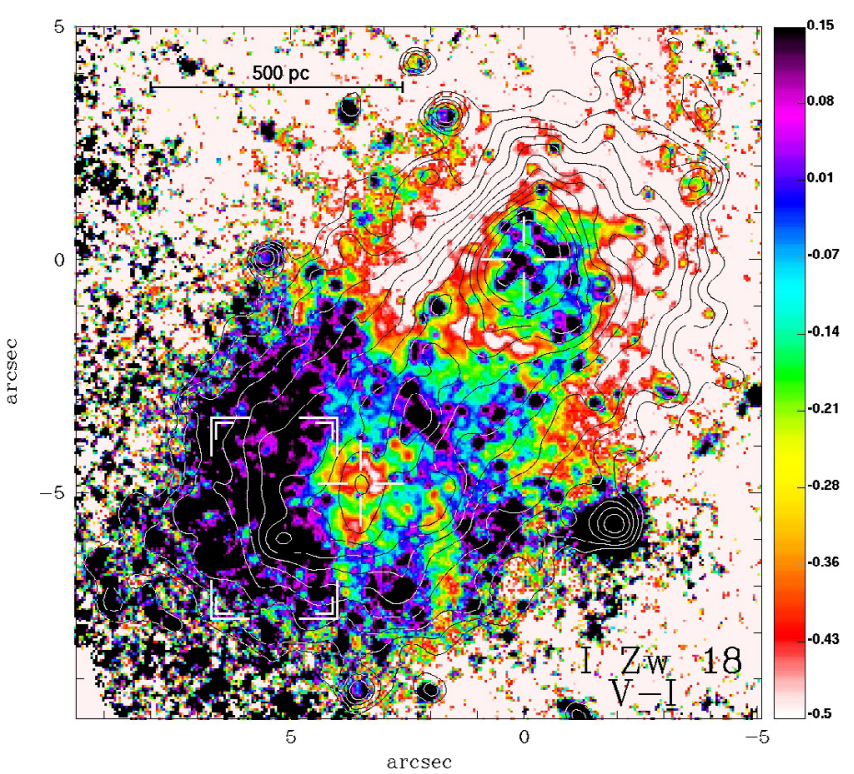

Fig. 7. $V-I$ map of the central 15 ". $^{\prime} \times 15^{\prime \prime} .6$ of I $\mathrm{Zw} 18$, displayed in the color range between -0.5 and 0.15 mag. The contours are computed from the $R^{\prime}$ image by P02 (see also Fig. 8) and go from 19 to $25 \mathrm{mag} / \square^{\prime \prime}$ in steps of $0.5 \mathrm{mag}$. Note the very blue $(<-0.5 \mathrm{mag})$ rim encompassing the $\mathrm{SF}$ region NW.

to be dominated by stars. More impressively, at $R^{\star} \sim 6^{\prime \prime}$, i.e. roughly at the transition radius between the HSB core and the exponential LSB envelope of SBPs, either color index shows a sudden decrease to values as blue as $V-I=-0.61 \pm 0.13 \mathrm{mag}$ and $R-I=-1.1 \pm 0.08 \mathrm{mag}$, which then remain nearly constant out to $R^{\star} \sim 20^{\prime \prime}$.
That such colors cannot be of stellar origin is apparent already from the fact that, even for an $\mathrm{O} 5 \mathrm{~V}$ star, the $V-I$ color is $-0.32 \mathrm{mag}$ and $R-I$ is $-0.18 \mathrm{mag}$, i.e. 0.3 to $0.9 \mathrm{mag}$ redder than the color of the LSB envelope of I Zw 18. More generally, as pointed out by $\mathrm{P} 02$, there is no stellar population, regardless of star formation history, age, and metallicity, that can reproduce the observed combination of red $(\sim 0.6 \mathrm{mag}) V-R$ with blue $(-0.6 \ldots-1.1 \mathrm{mag}) V-I$ and $R-I$ colors of the LSB envelope of I Zw 18. Quite the contrary, such colors can be accounted for solely by ne.

Indeed, comparison of our Fig. 5 and Fig. 12 of P02 reveals that the radius $R^{\star}=6^{\prime \prime}-7^{\prime \prime}$ where the steep $V-I$ and $R-I$ color drop-off occurs is identifiable with the radius where the line-of-sight contribution of stellar emission steeply decreases from a plateau value of $\sim 40 \%$ for $3 \lesssim R^{\star} \lesssim 6^{\prime \prime}$ to less than $20 \%$. Therefore, the much deeper data studied here confirm and strengthen the previous conclusion that the extended $\left(6^{\prime \prime} \lesssim R^{\star} \lesssim 20^{\prime \prime}\right)$ exponential LSB envelope of I Zw 18 is due to ne.

The gaseous nature of the LSB envelope of I Zw 18 is also evident from the color maps in Figs. 6 and 7. These show a clear correspondence to radial color profiles, most notably a strong core-envelope color contrast $(0.5-1.5 \mathrm{mag})$ and remarkably uniform colors for the envelope $(V-R \sim 0.5 \ldots 0.6$, $R-I \approx-1.4 \mathrm{mag}, V-I \approx-1 \mathrm{mag}$ ) on a spatial scale of $9 \mathrm{kpc}^{2}$. Though prominent on direct images (cf. Fig. 1), the northwestern supershell, for example, is barely distinguishable from its gaseous surroundings on $V-I$ and $R-I$ color maps, suggesting a nearly constant spectral energy distribution (SED) over the full LSB envelope.

The relative extent of the stellar component that is confined to the compact HSB core with respect to the nebular LSB envelope is illustrated better in Fig. 8. The image reveals a complex network of ionized gas filaments extending as far out as 


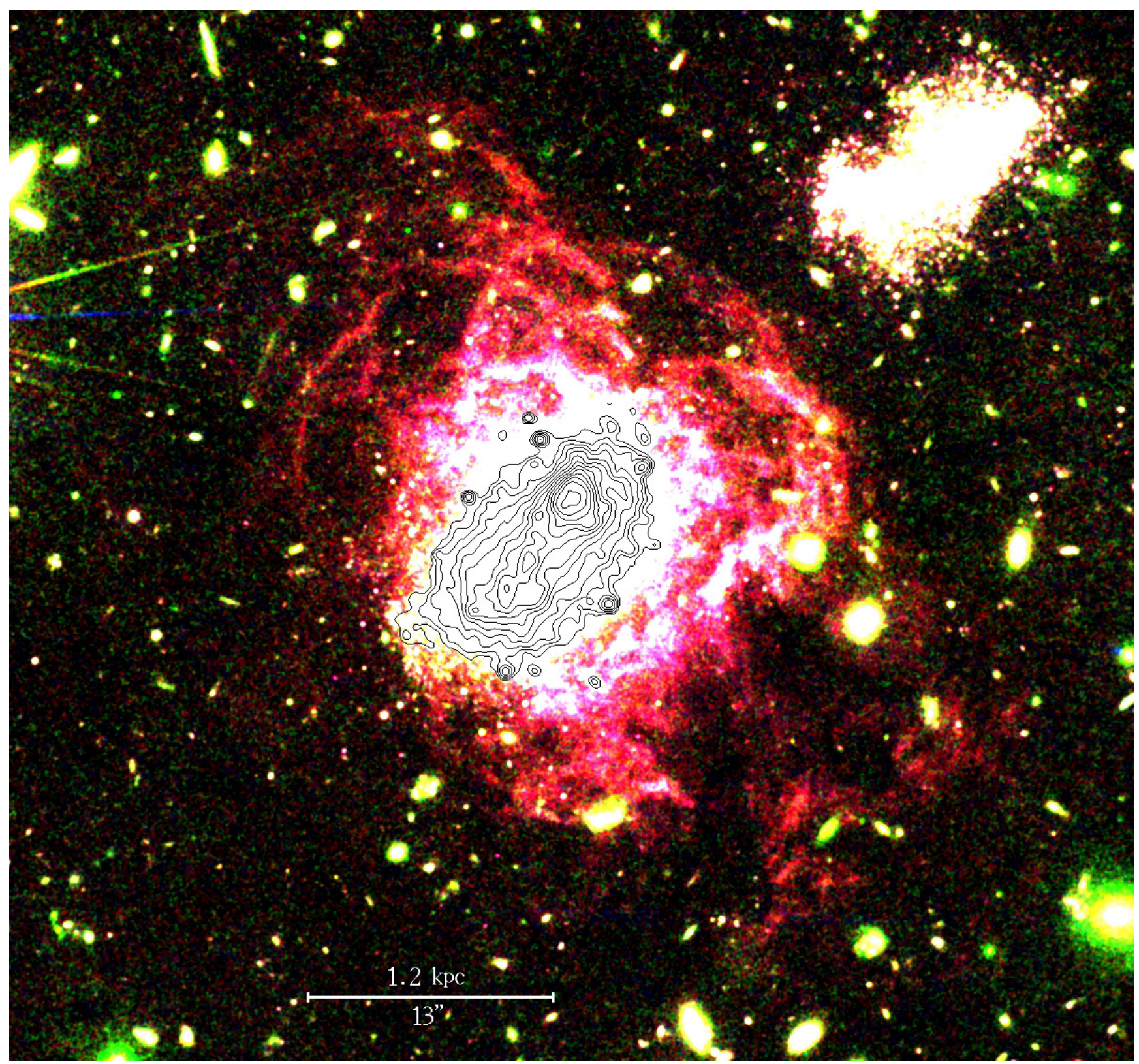

Fig. 8. Composite image of I Zw 18 and I Zw $18 \mathrm{C}$ with the $R, I$, and $V$ bands shown in the red, green, and blue channels, respectively. The contours, adapted from P02, are computed from HST WFPC2 $R$ data, after 2D subtraction of the $\mathrm{H} \alpha$ emission (referred to as $R^{\prime}$ ), they thus delineate the morphology of the stellar and nebular continuum emission in the main body of $\mathrm{I} \mathrm{Zw} 18$. Contours go from 19 to $25 R^{\prime}$ in steps of $0.5 \mathrm{mag}$.

$2.6 \mathrm{kpc}$, twice the galactocentric distance previously reported from HST WFPC2 studies and equivalent to $\sim 16$ exponential scale lengths $\alpha$ of the stellar host of I Zw 18 (160 pc; P02). The contours, adapted from P02, are computed from HST WFPC2 $R$ data, after $2 \mathrm{D}$ subtraction of $\mathrm{H} \alpha$ line emission (referred to as $R^{\prime}$ ), they thus delineate the morphology of the stellar and nebular continuum emission. I ZW 18 (i.e. its stellar component as depicted by the $R^{\prime}$ contours) and I Zw $18 \mathrm{C}$ show remarkably similar structures.

\subsubsection{The impact of nebular emission on the colors of the stellar component of I Zw 18}

We discuss next the effect of ne contamination in the HSB core in more detail, i.e. the region subtended by the $25 R^{\prime} \mathrm{mag} / \square^{\prime \prime}$ isophote in Fig. 8. Our color maps reveal here a substantial substructure that was not sufficiently recovered from previous data. A previously known feature is the ne $\operatorname{rim}(E W(\mathrm{H} \alpha)=$ 1500-2000 Å; Östlin et al. 1996; Vílchez \& Iglesias-Páramo 1998; Papaderos et al. 2001; Izotov et al. 2001a) that encompasses the SF region NW. Its $V-I$ and $R-I$ colors are much bluer ( $-0.8 \mathrm{mag}$ and $-0.6 \mathrm{mag}$, respectively) than those of the centrally located young SCs $(-0.15 \mathrm{mag}$ and $-0.23 \mathrm{mag}$, measured within a 2 .' $8 \times 2$ '. 8 aperture) where the $E W(\mathrm{H} \alpha)$ is much lower ( 200 ̊; Izotov et al. 2001a).

This striking spatial anticorrelation between stellar surface density and emission-line $E W \mathrm{~s}$, with the $V-I$ color steeply decreasing with the increasing $E W$ in the periphery of ionizing SCs is essentially identical to what is described in the XBCD SBS 0335-052E by P98: the bluest $V-I$ colors $(-0.8 \mathrm{mag})$ in that galaxy were not observed at the position of its young SCs but in their periphery, over an extended horseshoe-shaped gaseous 


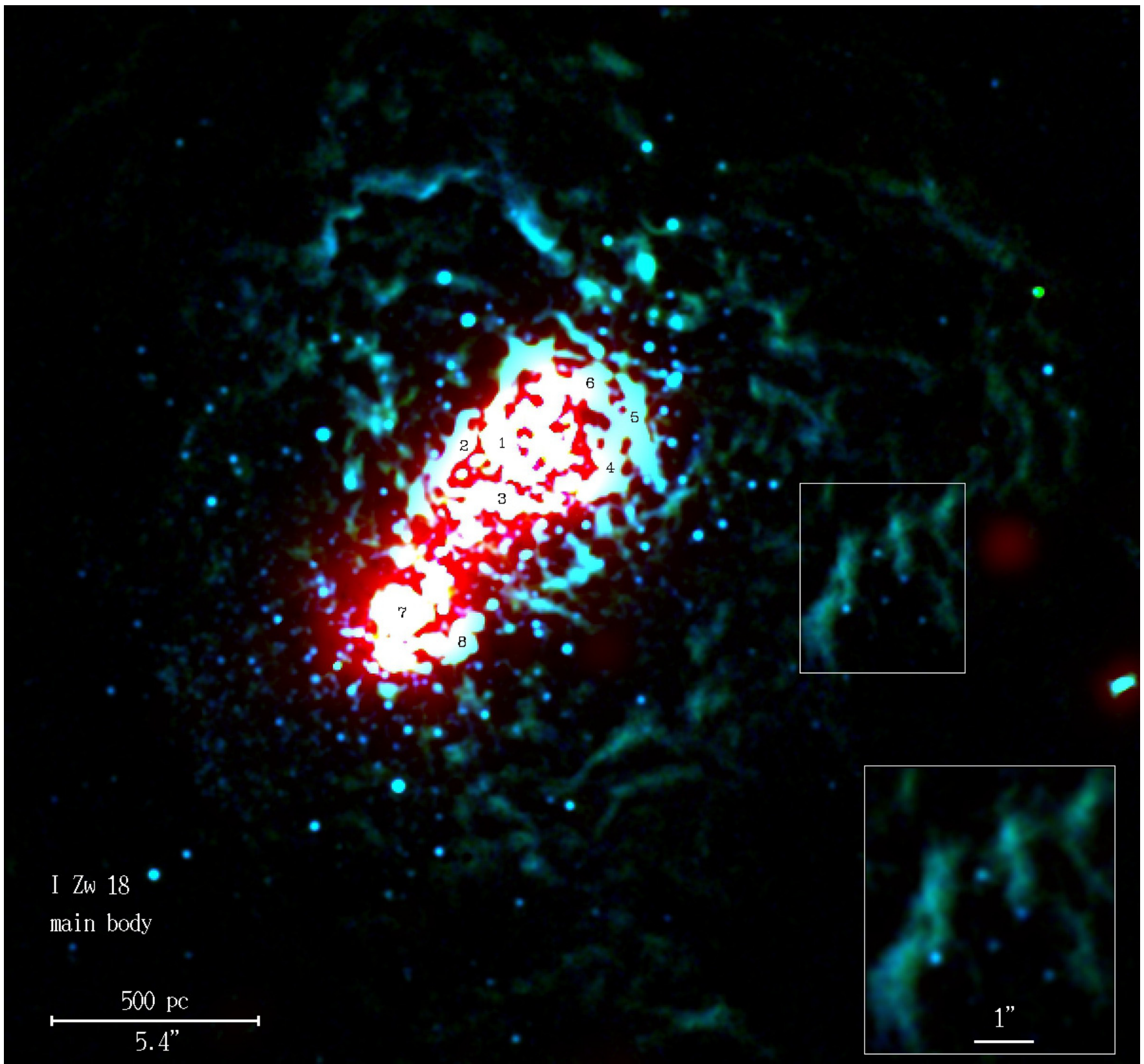

Fig. 9. Three-color image of I Zw 18 displaying the unresolved stellar emission $\left(I_{\mathrm{d}}\right)$ in the red channel and the $R$ and $V$ emission of compact ne-dominated regions in the green and blue channels. White areas in the central (red) part of the galaxy depict regions whose colors are strongly affected by nebular emission, as is apparent from their extremely blue $V-I$ and $R-I(<-0.4 \ldots-0.8)$ colors. The inset, showing a magnified portion of the western supershell, is meant to illustrate a subtle spatial shift between the $V_{\mathrm{c}}$ and $R_{\mathrm{c}}$ emission. This can be attributed to a spatial displacement by $30-100 \mathrm{pc}$ between the intensity maxima of the [ОІІІ] $\lambda \lambda 4959,5007$ and $\mathrm{H} \alpha$ emission lines.

rim some $500 \mathrm{pc}$ offset from the former. Several similar examples are documented among XBCDs and BCDs (e.g. Papaderos et al. 1999; Guseva et al. 2001; Fricke et al. 2001; Östlin et al. 2003; Guseva et al. 2004; Papaderos et al. 2008) both on small and large spatial scales.

Of considerable interest is also the small-scale contamination of colors in the HSB core of I Zw 18 due to ne. To better illustrate its impact, we computed a pseudo three-color composite (Fig. 9) using a combination of unsharp masked images (see discussion in Sect. 3.1). To partly suppress the stellar contribution in $V_{\mathrm{c}}$ and $R_{\mathrm{c}}$ images (blue and green channels, respectively), we subtracted from the latter a pseudo stellar continuum using a scaled version of the $I_{\mathrm{c}}$ image. This procedure allowed us to better isolate and visualize regions where ne contamination is strongest. The red channel in Fig. 9 holds the unresolved emission in the $I$ band $\left(I_{\mathrm{d}}\right)$ and thus primarily reflects the stellar host galaxy. In the resulting three-color overlay, white patches on top of the reddish background of the HSB core depict regions where ne dictates colors. Just like in radial profiles and color maps (Figs. 5-7), the footprint of ne is a combination of red $V-R$ with extremely blue $V-I$ and $R-I$ colors. Some examples include the regions labeled 1 through 8 , whose respective colors are $(0.1,-0.35,-0.45),(0.17,-0.58,-0.76),(0.05,-0.41$, $-0.45),(0.23,-0.43,-0.67),(0.24,-0.45,-0.69),(0.21,-0.53$, $-0.74),(0.12,-0.35,-0.47)$, and $(0.23,-0.23,-0.46)$. The total area of these severely ne-contaminated regions of $\approx 30 \square^{\prime \prime}$ is 


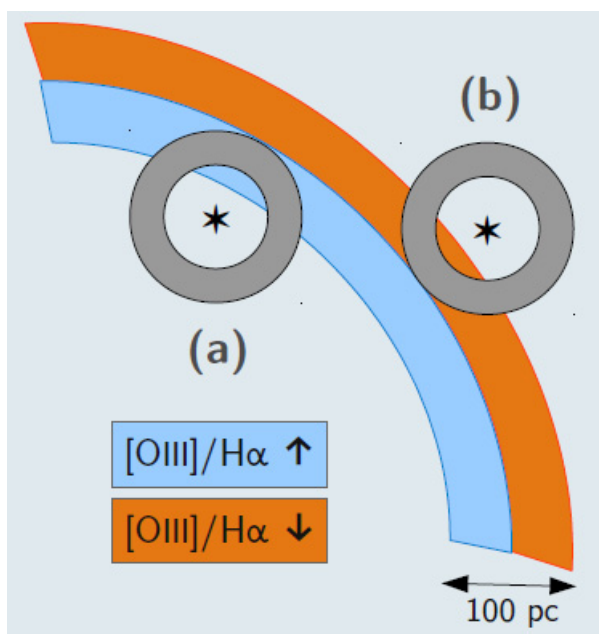

Fig. 10. Schematic illustration of the effect that the slight spatial displacement between the [OIII] $\lambda \lambda 4959,5007$ and the $\mathrm{H} \alpha$ emission lines across an expanding shell of ionized gas might have on the $V$ and $R$ photometry of stars in its close vicinity. The shell, of thickness $100 \mathrm{pc}$ $\left(\approx 1^{\prime \prime}\right.$ at the distance to I $\left.\mathrm{Zw} 18\right)$, comprises an inner and outer zone with, respectively, higher and lower [OIII]/H $\alpha$ ratios (cf. the discussion related to Figs. 6 and 9).

equivalent to the isophotal size of I Zw 18 at $22 \mathrm{mag} / \square^{\prime \prime}$ (32 $\square^{\prime \prime}$ ) and to $20 \%$ of that of its stellar component (the region subtended by the $25 R^{\prime} \mathrm{mag} / \square^{\prime \prime}$ isophote in Fig. 8).

It is noteworthy that ne shows significant substructure on spatial scales of a few pixels, with little spatial correlation to the local stellar background. Therefore, its treatment and subtraction as a uniform foreground emitting layer in CMD studies could result in systematic uncertainties that might not be fully accounted for by the standard error budget. Subtle spatial displacements between the intensity maxima of strong nebular emission lines may be relevant in this regard. An example is given in the inset of Fig. 9 where we show magnified a portion of the western supershell. It can be seen that the inner layer along the shell appears blueish whereas the outer one greenish. This can be plausibly attributed to a displacement by $0{ }^{\prime} .1-0.3$ between the [OIII] $\lambda \lambda 4959,5007$ and $\mathrm{H} \alpha$ emission lines, which are registered in $V$ (blue channel) and $R$ (green channel), respectively. Another illustrative example is given for a shell $\sim 5^{\prime \prime}$ northwards of region NW (Fig. 6) whose inner (southeastern) and outer (northwestern) layer differ by $\approx 0.3$ mag in their $V-R$ color, pointing to a variation by $\gtrsim 30 \%$ in the [OIII] $/ \mathrm{H} \alpha$ ratio across the shell. Systematic variations of the $[\mathrm{O}$ III $] \lambda 5007 / \mathrm{H} \beta$ ratio by a factor $\sim 3$ on spatial scales of a few ten pc have been documented by integral field unit spectroscopy in a number of Hir regions and BCDs (see e.g. Westmoquette et al. 2007; Cairós et al. 2009; Relano et al. 2010; Monreal et al. 2010).

Whereas spatial displacements between nebular lines certainly have no measurable effect on CMD studies of galaxies with faint ne, they may be important in the case of I Zw 18. This is because the local background level in point source photometry studies may be overestimated by a different amount in different bands, depending on the position of a star relative to a shell and the specifics of the local background determination. Figure 10 illustrates two special cases schematically: for a star located near the inner interface of the shell (case a), the circular annulus within which the local background is determined captures the inner [OIII]-enhanced portion of the shell and misses its outer $\mathrm{H} \alpha$-enhanced layer. This could lead to an over-subtraction of the local $V$ background, hence to a reddening of the $V-R$ color. The opposite might be the case for a star close to the outer boundary of the shell (case b), whose color would appear bluer due to the overestimation of the $R$ ( $\mathrm{H} \alpha$ enhanced) background. In the simple geometry of Fig. 10, the principal effect would then be a redder color for stars in the shell interior and vice versa, in addition to underestimated stellar magnitudes because of oversubtraction of the local background. An examination of the cumulative effect that multiple overlapping ne shells could have on CMD studies of I Zw 18 might therefore be of some interest.

Of special relevance to the study of the evolutionary status of I Zw 18 (Sect. 4.2) are the colors of region $\omega$ (Fig. 1). This region, roughly delimited by the rectangular area at (RA,

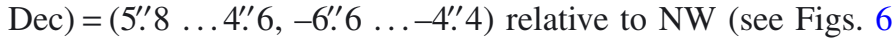
and 7) is relatively free of ne contamination (cf. the $E W(\mathrm{H} \alpha)$ map in Fig. 7 of Izotov et al. 2001a), its colors can therefore be used to place constraints on the age of I Zw 18. From the present data we infer a mean $V-R$ color of -0.06 mag and a $R-I$ and $V-I$ color of $\approx 0.2$ mag with values of $V-R \simeq R-I \approx 0.15$ mag and $V-I=0.3 \mathrm{mag}$ within its reddest quarter. The $V-I$ color range inferred for region $\omega$ from HST ACS data is in good agreement with the values $0.17-0.32$ mag previously determined by P02.

\section{Discussion}

\subsection{Constraints on the evolutionary status of I Zw $18 \mathrm{C}$}

In Fig. 11 we compare the observed colors of I $\mathrm{Zw} 18 \mathrm{C}$ with the predicted color evolution for a stellar population forming instantaneously (SFH1), with an exponentially decreasing star formation rate (SFR) and an e-folding time $\tau=1 \mathrm{Gyr}$ (SFH2) and continuously with a constant SFR (SFH3). The theoretical curves were computed with the evolutionary synthesis code Pegase 2.0 (Fioc \& Rocca-Volmerange 1997) for constant stellar metallicity of $Z=0.001$ and Salpeter initial mass function (IMF) between 0.1 and $100 M_{\odot}$, and do not include nebular emission.

As already pointed out in Sect. 3.1, the blue and nearly constant $(0 \pm 0.05 \mathrm{mag}) V-I$ and $R-I$ colors of I Zw $18 \mathrm{C}$ down to $27.6 \mathrm{mag} / \square^{\prime \prime}$ imply that the photometrically dominant stellar population in this system is almost uniformly young. Formal upper age estimates within $1 \sigma$ uncertainties range from $t_{1}=15 \mathrm{Myr}$ for SFH1 to $t_{2} \sim 35 \mathrm{Myr}$ for SFH2,3. The last two SF scenarios are, however, incompatible with the data since star formation starting at $t_{2}$ and continuing to the present would have given rise to ample ne with an $E W(\mathrm{H} \alpha) \gtrsim 800 \AA$. The uniformly blue colors of I $\mathrm{Zw} 18 \mathrm{C}$, in connection with the absence of ne everywhere but in its central region indicate that $\mathrm{SF}$ activities must have ceased very recently, some $\sim 20 \mathrm{Myr}\left(=t_{\mathrm{c}}\right)$ ago. If so, the estimated SF duration $\left(t_{2}-t_{\mathrm{c}} \simeq t_{1}\right)$ would imply an almost instantaneous SF process, rapidly synchronized over the projected area of the galaxy $\left(\sim 1 \mathrm{kpc}^{2}\right)$ probably at supersonic speeds.

A conceivable scenario might invoke sequential star formation from the redder northwestern tip towards the bluer southeastern tip of I Zw 18 C (see also Aloisi et al. 1999; Izotov et al. 2001a). The respective colors of those regions translate by SFH1 to an age difference of $\tau_{\mathrm{SF}}=40-80 \mathrm{Myr}$. Taking $\tau_{\mathrm{SF}} \sim 60 \mathrm{Myr}$ as an indicative timescale for the spatial progression of SF activities along the projected major axis of I ZW $18 \mathrm{C}(1 \mathrm{kpc})$, one can estimate an average SF propagation velocity of $u_{\mathrm{SF}} \sim 20 \mathrm{~km} \mathrm{~s}^{-1}$. This is comparable to the $u_{\mathrm{SF}}$ inferred for the XBCD SBS 0335 052E ( 20-35 km s${ }^{-1}$, P98, Reines et al. 2008), close to the sound speed in the warm ISM. Induced gas collapse along the collisional interface of a supershell expanding from the northwestern tip of I Zw $18 \mathrm{C}$ between $80 \mathrm{Myr}\left(\tau_{\mathrm{SF}}+t_{\mathrm{c}}\right)$ and $t_{\mathrm{c}}$ ago 


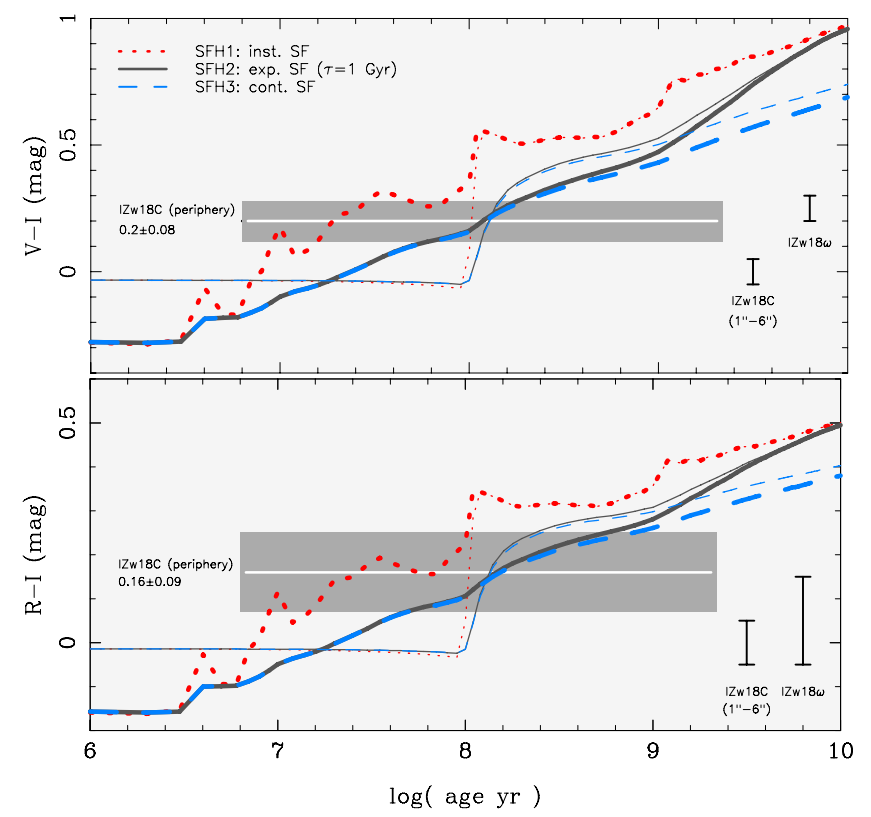

Fig. 11. Comparison of the $V-I$ and $R-I$ colors of $\mathrm{I} \mathrm{Zw} 18 \mathrm{C}$ and of region $\omega$ in $\mathrm{I} Z \mathrm{~W} 18$ with model predictions for a stellar population forming instantaneously (SFH1: thick dotted curve) or continuously, with an exponentially decreasing or constant star formation rate (SFH2 and SFH3: thick solid and dashed lines, respectively). The models have been computed with Pegase 2.0 (Fioc \& Rocca-Volmerange 1997) for a constant metallicity $(Z=0.001)$ and a Salpeter initial mass function between 0.1 and $100 M_{\odot}$, and do not include nebular emission. Thin curves correspond to the same star formation histories but assume an IMF truncated above $5 M_{\odot}$. The vertical bars labeled IZw $18 \omega$ depict the range between the mean and reddest color in region $\omega$ of I Zw $18(V-I=0.2$ $\ldots 0.3$ and $R-I=-0.06 \ldots 0.15)$. Bars labeled I Zw $18 \mathrm{C}\left(1^{\prime \prime}-6^{\prime \prime}\right)$ indicate the mean color of $\mathrm{I} \mathrm{Zw} 18 \mathrm{C}$ in the radius range $1^{\prime \prime} \leq R^{\star} \leq 6^{\prime \prime}$ $(\approx 0 \pm 0.05 \mathrm{mag})$. The mean colors and their $1 \sigma$ uncertainties in the extreme periphery $\left(R^{\star} \geq 6^{\prime \prime}\right)$ of I Zw $18 \mathrm{C}$ at $\mu \gtrsim 27.6 \mathrm{mag} / \square^{\prime \prime}$ are depicted by the rectangular areas.

might offer a tenable, though not necessarily unique, explanation for propagating star formation. Following McCray \& Kafatos (1987), the radius $R_{\mathrm{sh}}$ (pc) of a SF-driven supershell can be approximated as

$R_{\mathrm{sh}}=269\left(\frac{L_{\mathrm{m}, 38}}{n_{0}}\right)^{1 / 5} t_{7}^{3 / 5}$

where $L_{\mathrm{m}, 38}$ is the mechanical luminosity injected into the ISM by stellar winds and SNe in $10^{38} \mathrm{erg} \mathrm{s}^{-1}, n_{0}$ the ambient gas density in $\mathrm{cm}^{-3}$ and $t_{7}$ the dynamical expansion time in $10^{7} \mathrm{yr}$. A rough estimate of the mean mechanical luminosity can be inferred from the absolute $V$ magnitude of I Zw $18 \mathrm{C}$ ( $-12.2 \mathrm{mag})$, which for continuous star formation over $\tau_{\mathrm{SF}}$ and an ensuing quiescent phase over the past $t_{c}$ translates into a stellar mass of $\approx 2.1 \times 10^{5} M_{\odot}$ and a mean SFR of $3.5 \times 10^{-3} M_{\odot} \mathrm{yr}^{-1}$. The mean $L_{\mathrm{m}, 38}$ over $\tau_{\mathrm{SF}}$ may be estimated from Starburst 99 (Leitherer et al. 1999) to be 14.6. Equation (3) then yields an ambient gas density $n_{0} \approx 4 \mathrm{~cm}^{-3}$ for $R_{\mathrm{sh}}=1 \mathrm{kpc}$ and $t_{7}=6$ $\left(\equiv \tau_{\mathrm{SF}}\right)$. This value does not seem to be unrealistic, given that the VLA HI map by van Zee et al. (1998) indicates an Hi surface density of $\gtrsim 4 \times 10^{20} \mathrm{~cm}^{-2}$ for I Zw $18 \mathrm{C}$, which after including the helium contribution translates to $n_{0} \sim 3 \mathrm{~cm}^{-3}$ for a disk thickness of $\sim 50 \mathrm{pc}$. Because the passage of the SF front along I Zw $18 \mathrm{C}$ has probably been accompanied by the depletion of its molecular content and the dispersal of its ISM, the present gas surface density should, in fact, represent a lower estimate for the average $n_{0}$ over $\tau_{\mathrm{SF}}$. Clearly, the considerations above rely on strongly simplifying assumptions (constant $L_{\mathrm{m}, 38}$ and $n_{0}$, no radiative losses in the shell, coplanar face-on geometry) and are only meant to be indicative. They merely touch upon one among several possible scenarios behind propagating star formation in I Zw $18 \mathrm{C}$ (e.g., gravitational interaction with the main body or gas infall from the Hi halo) and are invoked to demonstrate that this process can account for some important characteristics of the galaxy. These include its uniformly blue colors with merely a weak color gradient along its major axis, the virtual absence of ne, and the higher surface density of the unresolved stellar background in its northwestern half as a possible signature of the disintegration of its oldest SCs. However, propagating star formation between $\tau_{\mathrm{SF}}+t_{\mathrm{c}}$ and $t_{\mathrm{c}}$ cannot alone explain the slightly redder color of stellar populations on the outskirts of I Zw $18 \mathrm{C}$.

\subsubsection{Stellar diffusion and its effect on age determinations in the faint periphery of I Zw $18 \mathrm{C}$}

In the following we explore the evolutionary history of I $\mathrm{ZW} 18 \mathrm{C}$ further by considering the properties of its faint $\left(27.6-29 \mathrm{mag} / \square^{\prime \prime}\right)$ stellar periphery. The $(V-I)_{\mathrm{d}}(0.2 \pm 0.08)$ and $R-I(0.16 \pm 0.09)$ color of the latter translate by SFH2 to an age of $\sim 130 \mathrm{Myr}$ with an upper bound of $\sim 500 \mathrm{Myr}$ at the $1 \sigma$ level, if purely stellar emission is assumed. For models including ne, age estimates would rise to 270-900 Myr, depending on the color considered. However, even for those high ages, this $\mathrm{SFH}$ model (and SFH3 alike) predicts an $E W(\mathrm{H} \alpha)$ between $150 \AA$ and $\gtrsim 300 \AA$, in clear conflict with the absence of ne in the outskirts of I Zw $18 \mathrm{C}$. Models invoking continuous star formation to the present are thus fundamentally incompatible with the data. Cessation of SF activities over the past $t_{\mathrm{c}}$ would alleviate the contradiction; however, it would at the same time imply a steeper color evolution, thus a younger age, in better agreement with the $1 \sigma$ upper estimate $t_{\mathrm{SFH} 1} \sim 100 \mathrm{Myr}$ read off Fig. 11 for the instantaneous star formation model.

As we argue next, stellar diffusion and the resulting radial stellar mass filtering effect described by P02 could have a significant effect on the color distribution of young galaxy candidates and add an important element towards a consistent evolutionary picture for I $\mathrm{Zw} 18 \mathrm{C}$. Already at a mean radial velocity of $u_{\mathrm{r}} \lesssim 4 \mathrm{~km} \mathrm{~s}^{-1}$, less than the velocity dispersion of the neutral gas $\left(\sigma_{\mathrm{HI}} \approx 6-8 \mathrm{~km} \mathrm{~s}^{-1}\right)$, a star born in the central part of I Zw $18 \mathrm{C}$ could migrate within $\tau_{\text {diff }}=\tau_{\mathrm{SF}}+t_{\mathrm{c}}(80 \mathrm{Myr})$ out to $r_{\mathrm{diff}} \approx 300 \mathrm{pc}$ from its initial locus. This galactocentric distance corresponds to the semi-minor axis of I Zw $18 \mathrm{C}$ at $29 \mathrm{mag} / \square^{\prime \prime}$; i.e., it is topologically identifiable with the redder, outer exponential SBP feature in Fig. 3. A consequence of stellar diffusion with the above radial velocity is that any stellar generation reaching out to $r_{\text {diff }}$ cannot be bluer than $0.3 \mathrm{mag}$ in $V-I$ (approximately the $1 \sigma$ color bound, depicted by the rectangular area in the upper panel of Fig. 11), since on its way to that radius it will have been depopulated from stars with lifetimes $\leq \tau_{\text {diff }}$ (or, correspondingly, from stars more massive than $M_{\text {diff }}$ ). The commonly adopted SFH2,3 models would then inevitably result in an overestimation of the stellar age in the LSB periphery $\left(R^{\star} \simeq r_{\text {diff }}\right)$ of young galaxy candidates, such as I Zw $18 \mathrm{C}$. This is because such $\mathrm{SFH}$ parametrizations are applied throughout on the implicit assumption that stellar populations form in the radial zone where they are currently observed, so they invariably contain a certain fraction of massive blue stars younger than $\tau_{\text {diff. }}$ Obviously, a match between predicted and observed colors is then only possible for 
an age $t_{\mathrm{SFH} 2,3}$ exceeding the true stellar age $t_{\star}$ at the radius $R^{\star}$; i.e., the condition

$\tau_{\text {diff }} \leq t_{\mathrm{SFH} 1} \leq t_{\star}<t_{\mathrm{SFH} 2,3}$

applies throughout, in particular when models including ne are adopted.

That, in the presence of stellar diffusion, the age of the LSB host of young BCD candidates may be overestimated when continuous star formation models are used is especially apparent when projection effects are additionally taken into account: in a spherical-symmetric geometry, the colors registered at $R^{\star} \equiv r_{\text {diff }}$ reflect the luminosity-weighted average of stars at radii $\geq r_{\text {diff }}$, since the observer's line of sight crosses more remote foreground and background galaxy zones of longer $\tau_{\text {diff }}$. Therefore, even though SFH2,3 may provide a reasonable approximation to the integral SFH of some SF dwarf galaxies (SFDGs), it should not be taken for granted that they are universally applicable to spatially resolved age-dating studies. This is already suggested by the failure of, e.g., SFH3 to reproduce the generally red colors ( $V-I=0.9, B-R=1.1$ ) of the host galaxy of typical BCDs (see e.g. P02), even for ages exceeding the Hubble time.

The effect of stellar diffusion to the radius $r_{\text {diff }}$ is equivalent to in situ star formation at $r_{\text {diff }}$ with an IMF that is truncated at masses above a radially decreasing limit $M_{\text {diff }}\left(\tau_{\text {diff }}\right)$ (hereafter tIMF). To illustrate this point, and as a minimum consistency check, one may consider continuous star formation according to SFH2,3 with a tIMF limited to stars with a lifetime $\gtrsim \tau_{\text {diff }}$. This timescale roughly corresponds to the main sequence lifetime of a B4 star (94 Myr), placing an upper cutoff of $5 M_{\odot}$ to the tIMF. The color evolution for SFH2,3 with that tIMF is depicted in Fig. 11. It can be seen that SFH2,3+tIMF models reproduce the observed colors at an age of $\sim 130$ Myr with an $1 \sigma$ upper bound of 150-250 Myr and consistently account for the absence of nebular emission.

A conceivable alternative to stellar diffusion is in situ star formation in the LSB periphery of I Zw $18 \mathrm{C}$ in conjunction with stochastic effects on the IMF (see Cerviño \& Mas-Hesse 1994, and references therein). The latter are to be expected when low masses are involved in the SF process, as is typically the case for SFDGs, and to affect the sampling of the IMF in its intermediateto-high mass range. Cerviño \& Mas-Hesse (see their Fig. 1) argue, however, that stochastic effects do not result in a truncated IMF above a certain stellar mass, but some massive stars always form. On the statistical average, one would therefore expect nebular emission to be present in the outskirts of I $\mathrm{ZW} 18 \mathrm{C}$, in disagreement with the observations. It should be noted, though, that this conclusion very likely depends on the detailed physics of star formation, from which the high-mass end of the IMF is populated and the timescales for low- and high-mass star formation. With certain assumptions (see e.g. Weidner \& Kroupa 2005; Pflamm-Altenburg et al. 2009), a truncated IMF can be realized in peripheral galaxy zones, lifting the discrepancy described above. There might therefore be variants of the traditional static scenarios, i.e. scenarios implicitly assuming that stars are born in the radial zone where they are observed, which can quantitatively reproduce the radial color distribution of I $\mathrm{Zw} 18 \mathrm{C}$ and SFDGs in general. It is not clear, however, whether such static scenarios, and their assumptions on the radial dependence of the IMF, can consistently account for the specific structural characteristics of SFDGs, and in particular BCDs, such as the exponentiality of the stellar LSB host and the confinement of SF activities to within $R^{\star} \approx 2 \alpha$ (see e.g. P96b).

Stellar diffusion and its role in the buildup of SFDGs constitutes an unexplored territory in dwarf galaxy research. A caveat of the above discussion is that it assumes young stars to initially drift apart freely at a roughly constant velocity $u_{\mathrm{r}}\left(\lessgtr \sigma_{\mathrm{HI}}\right)$ over $\sim 10^{8}$ yr. Clearly, the validity of this simplifying assumption needs to be investigated, and the initial kinematics of the newly formed stars, as well as the form of the gravitational potential should be included in the analysis. High-velocity resolution ( $\$ 10 \mathrm{~km} \mathrm{~s}^{-1}$ ), integral field spectroscopy might provide key insights into the velocity patterns and the possible outwards migration of bound and unbound stellar clusters in young starbursts. A closer investigation of the effects of diffusion would be of considerable interest, among others, because it offers a mechanism that naturally produces radial color gradients in galaxies, which might be relevant to e.g. the Red Halo phenomenon (Bergvall \& Östlin 2002; Zackrisson et al. 2006; Bergvall et al. 2010).

In summary, our results suggest that the overall photometric properties of I Zw $18 \mathrm{C}$ can be consistently accounted for by a single episode involving solely sequential star formation $\left(u_{\mathrm{SF}} \approx\right.$ $\left.20 \mathrm{~km} \mathrm{~s}^{-1}\right)$ and stellar diffusion $\left(u_{\mathrm{r}} \approx 4 \mathrm{~km} \mathrm{~s}^{-1}\right)$ over the past 100-200 Myr. Whereas an ultra-faint substrate of ancient stars cannot be ruled out per se, the lines of evidence presented here indicate that $\mathrm{I} \mathrm{Zw} 18 \mathrm{C}$ is a cosmologically young object. This conclusion concurs with the one of Izotov et al. (2001a) who infer an upper age of $\sim 100 \mathrm{Myr}$ for I Zw $18 \mathrm{C}$ from spectral synthesis models. Jamet et al. (2010) reach a similar conclusion, that I $\mathrm{Zw} 18 \mathrm{C}$ is younger than $\approx 125 \mathrm{Myr}$, from a probabilistic analysis of HST ACS CMDs.

\subsection{Constraints on the evolutionary status of I Zw 18}

We turn next to the evolutionary status of I Zw 18's main body. The $V-I$ and $R-I$ color range in region $\omega$ (Fig. 11), translate by SFH2,3 to an age between $\sim 100$ and $\$ 250$ Myr. The possibility of I Zw 18 and I Zw $18 \mathrm{C}$ forming a co-evolving pair of dwarf galaxies that underwent a roughly synchronous strong recent evolution can not be ruled out.

By considering the reddest quartile of region $\omega$, one obtains from SFH2,3 an upper age of 500 Myr. The age span inferred from the present study is consistent with that obtained from the mean $B^{\prime}-V^{\prime}(0.09 \pm 0.04 \mathrm{mag})$ and $V^{\prime}-R^{\prime}(0.12 \pm 0.04 \mathrm{mag})$ for the stellar host galaxy of I Zw 18 after 2D subtraction of strong nebular lines from broad band images (P02). However, the latter emission-line free colors are likely to be slightly overestimated, since they do not include corrections for the red ( $B-V=0.34, V-R=0.64$, see e.g. Krüger et al. 1995) nebular continuum. Notwithstanding this fact, even when taken within their $+1 \sigma$ bounds at face value, they imply by SFH 2 an age of $\sim 0.8 \mathrm{Gyr}$, translating into a mass fraction of $\gtrsim 50 \%$ for stars younger than 0.5 Gyr. Evidently, this conclusion (see also Hunt et al. 2003) does not conflict with the presence of a small number $(\$ 20)$ of stars with ages between 0.5 and $\sim 1 \mathrm{Gyr}$ in CMDs (Aloisi et al. 1999; Östlin 2000; Östlin \& Mouhcine 2005). On the other hand, it is important to bear in mind that estimates of the mass fraction of young stars critically depend on the adopted SFH.

\subsubsection{An extended underlying stellar disk in I Zw 18?}

We next revisit the hypothesis envisaged by Legrand (2000), according to which the formation of I Zw 18 occurs throughout its Hi halo $\left(60^{\prime \prime} \times 45^{\prime \prime}\right)$ at an extremely low SFR over the past $\sim 14$ Gyr. This model can reconcile the low gas-phase metallicity of I Zw 18 with a cosmological age, and predicts a high degree of chemical homogeneity in its warm ISM. From the photometric 
point of view, the main prediction from the Legrand model is an extended ultra-LSB underlying stellar disk $\left(\bar{\mu} \simeq 28 \mathrm{~V} \mathrm{mag} / \square^{\prime \prime}\right)$. In a subsequent study, Legrand et al. (2001) generalize the scenario of "slowly cooking" dwarfs to BCDs, arguing that their main metallicity and stellar mass contributor is continuous star formation, rather than coeval starbursts.

The hypothesis of a stellar disk beneath the nebular envelope of I Zw 18 was investigated later on by $\mathrm{P} 02$ on the basis of emission-line free HST WFPC2 $B^{\prime}, V^{\prime}$, and $R^{\prime}$ images. By consideration of photometric uncertainties, $\mathrm{P} 02$ argue that the predicted disk would evade detection on their SBPs if its central surface brightness $\mu_{\mathrm{E}, 0}$ is fainter than $27.1 \mathrm{mag} / \square^{\prime \prime}$ and its exponential scale length $\alpha$ is larger than 10.5 . These limits translate to an apparent magnitude of $20 \mathrm{mag}$ and a $\bar{\mu} \simeq 28.6 \mathrm{mag} / \square^{\prime \prime}$, slightly fainter, but probably still consistent within the uncertainties, with the $\bar{\mu}$ predicted by the Legrand model. Thus, previous surface photometry could neither strictly rule out nor establish the presence of the putative ultra-LSB disk in I Zw 18. Since deep HST ACS narrow-band imagery is unavailable for I Zw 18, we cannot improve on the nebular line subtraction carried out by P02 and push previous $V^{\prime}$ and $R^{\prime}$ surface photometry to fainter levels.

However, CMD studies of I Zw 18 argue against the disk hypothesis, as none among them has revealed a uniform and extended population of RGB stars being cospatial with the nebular envelope, in sharp contrast to any evolved BCD studied as yet. Admittedly, because none among the published CMD studies for I Zw 18 fully appreciates the importance of ne contamination (except for that by IT04) and attempts a proper assessment of the systematic errors this can introduce, the robustness of the nondetection of RGB candidates in the LSB envelope cannot be evaluated currently, and it is not clear whether CMD studies can ever place firm constraints in this respect. The currently most convincing evidence for the absence of a substantial stellar background beneath the nebular envelope of I Zw 18 is based on the emission-line free HST images by P02 (see their discussion). Even if an ultra-LSB stellar disk complying with the photometric limits placed by P02 was present, it would probably not significantly alter the interpretation of youth for I Zw 18: for continuous SFR over $14 \mathrm{Gyr}$, the absolute $V$ magnitude of the predicted disk $(-11.4 \mathrm{mag})$ translates into a stellar mass of $\sim 5.6 \times 10^{6} \mathrm{M}_{\odot}$. The $V^{\prime}$ magnitude of the host galaxy of I Zw 18 (i.e. excluding regions NW and SE) of -13.8 mag (P02) translates by SFH2 for an age between 0.5 and 1 Gyr to a stellar mass of $\sim 11 \times 10^{6} M_{\odot}$, i.e. about twice that of the hypothetical ultra-LSB disk. Even in the extreme case of a 14 Gyr old instantaneous burst, the underlying disk would only be twice as heavy as the young population that has formed on a much shorter timescale. Consequently, the conclusion that I Zw 18 has formed most of its stellar mass at a late cosmic epoch still holds. It would be interesting to check whether the combined chemical output from these two stellar populations is consistent with the observed gas-phase metallicity in I Zw 18.

With regard to the proposed generalization of the "slowly cooking" scenario, it should be noted that the Legrand model faces difficulties in reproducing basic photometric properties of BCDs. Specifically, the host galaxy of typical BCDs is incompatible with an ultra-LSB disk and, on the contrary, its central stellar density $\left(\geq 1 M_{\odot} \mathrm{pc}^{-3}\right)$ exceeds by an order of magnitude that of dwarf irregulars or dwarf spheroidals (P96b). Similarly, the mean surface brightness of the BCD host is $\leq 24 \mathrm{Bmag} / \square^{\prime \prime}$ (e.g. P96b, Amorín et al. 2009), roughly 4 mag brighter than the model prediction. Additionally, as pointed out in Sect. 4.1.1, continuous star formation models are inconsistent with the red
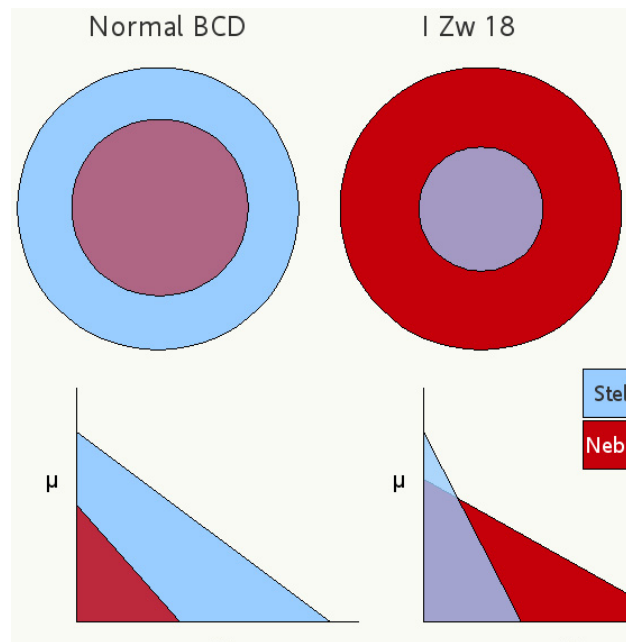

$\mathrm{R}^{*}$

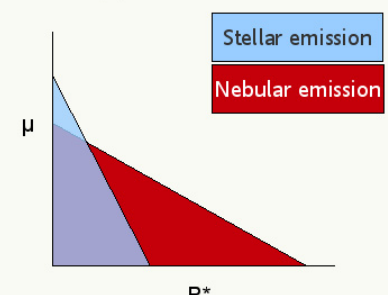

$R^{*}$

Fig. 12. Schematic comparison of typical blue compact dwarf (BCD) galaxies (left) with I Zw 18 (right) with respect to the morphology and radial intensity distribution (upper and lower figures, respectively) of their stellar and nebular emission.

host galaxy colors of most nearby BCDs. In summary, although appealing from the chemical point of view, the "slowly cooking" scenario does not seem to be reconcilable with the structural properties of BCDs. This scenario might, however, provide a good approximation of quiescent late-type dwarfs, such as blue low surface-brightness galaxies (Rönnback \& Bergvall 1994).

\subsection{Zw 18 as local morphological template for rapidly assembling galaxies at high redshift}

The integral photometric properties of the overwhelming majority of SF galaxies in the local universe are barely affected by ne. Typically, the $E W\left(\mathrm{H} \alpha+\left[\mathrm{N}_{\mathrm{II}}\right]\right)$ ranges between a few ten $\AA$ (e.g. Moustakas \& Kennicutt 2006; Koopmann \& Kenney 2006) in normal late-type galaxies to $\lesssim 10^{2} \AA$ for the majority of local SFDGs (see e.g. Lee et al. 2007; Sánchez Almeida et al. 2008). Even for BCDs, ne has a noticeable photometric impact $\left(E W\left(\mathrm{H} \alpha+\left[\mathrm{N}_{\mathrm{II}}\right]\right) \simeq 200-500 \AA\right.$; Terlevich et al. 1991; Cairós et al. 2002; Bergvall \& Östlin 2002; Gil de Paz et al. 2003; Guseva et al. 2009, among others) in their centrally confined SF component only (i.e. for $R^{\star} \lesssim R_{\mathrm{SF}}$, with $R_{\mathrm{SF}}$ on the order of $\sim 1 \mathrm{kpc}$ ) and is practically negligible for larger radii where the evolved stellar LSB host entirely dominates the line-of-sight intensity (P02, Knollmann 2004). More generally, even though ne in local SFDGs may protrude beyond $R_{\mathrm{SF}}$ (Hunter \& Gallagher 1985; Gallagher et al. 1989; Hunter \& Gallagher 1990, 1992; Meurer et al. 1992; Marlowe et al. 1995; Ferguson et al. 1996; Papaderos \& Fricke 1998; Martin 1998; Bomans 2002; Cairós et al. 2002, among others), its extremely low surface brightness means it has no impact on surface photometry (P02, Knollmann 2004). As an example, even for BCDs with strong ongoing SF activity, corrections of integral photometric quantities for ne do not exceed $\approx 0.1$ mag (Salzer et al. 1989).

In the nearby universe, the only cases of SFDGs with extreme ne contamination are documented in a few XBCDs. Intense and almost galaxy-wide SF activity in these rare young galaxy candidates, in combination with the low surface density of their underlying stellar host, boost [OIII] $\lambda 5007$ and $\mathrm{H} \alpha$ emission line $E W$ s to values of up to $\gtrsim 2 \times 10^{3} \AA$ and $\gtrsim 1.6 \times$ $10^{3} \AA$, respectively (e.g. P98, Izotov et al. $1997 \mathrm{a}$; Guseva et al. 2004; Izotov et al. 2006; Papaderos et al. 2006b, 2008, among 
others), i.e. close to the effective width of broad band filters. Similar, though less extreme, cases are some of the recently discovered ultra-compact starbursting dwarfs in galaxy clusters $\left(E W(\mathrm{H} \alpha) \sim 10^{3} \AA\right.$ A , Reverte et al. 2007).

As shown in $\mathrm{P} 02$, the radial $\mathrm{H} \alpha$ intensity profiles of BCDs comprise two characteristic components: an HSB core with $R^{\star} \simeq R_{\mathrm{SF}}$ and an outer, roughly exponential LSB envelope with $\alpha_{\mathrm{H} \alpha}$ in the range $0.1-1 \mathrm{kpc}$. Judging from its $\alpha_{\mathrm{H} \alpha}(210-270 \mathrm{pc})$ and radial extent $\left(R^{\star} \sim 2.6 \mathrm{kpc}\right)$, the ne halo of $\mathrm{I} \mathrm{Zw} 18$ is by no means exceptional among BCDs/SFDGs. However, I Zw 18 strikingly differs from any SFDG studied as yet because the galaxy itself (i.e. its stellar host, cf. Fig. 8) is several times more compact than the nebular halo: ne dominates already for $R^{\star} \simeq 6^{\prime \prime}$ ( $\equiv 3 R_{\text {eff }}$ ) and reaches as far out as $\sim 16$ stellar exponential scale lengths (Sect. 3.2). This so far unique case in the nearby universe is schematically illustrated in Fig. 12 where the radial distribution of stars and ne in I Zw 18 is compared with that of normal BCDs.

For the forthcoming discussion, it is specially important to recall that the surface brightness level at which in I $\mathrm{Zw} 18$ ne dominates is quite high $\left(\mu \simeq 23.5 \mathrm{mag} / \square^{\prime \prime}\right.$; cf. Fig. 5), i.e. comparable to the central surface brightness of dwarf irregulars (e.g. Patterson \& Thuan 1996; van Zee 2000) and dwarf ellipticals (Binggeli \& Cameron 1991, 1993), and by at least one mag brighter than that of Local Group dwarf spheroidals (cf. Mateo 1998). The ne envelope of I Zw 18 is therefore not to be confused with the extraordinarily diffuse ne in typical SFDGs. Also relevant is that the exponential ne envelope contributes at least one third of the total $R$ band luminosity of I Zw 18 (P02 and Sect. 3.2).

As we argue next, the morphological properties of I Zw 18, whereas unique among nearby SFDGs, are probably typical of rapidly assembling galaxies in the distant universe. Just like I Zw 18, these systems are building up their stellar component through starbursts or prolonged phases of strongly elevated specific SFR (SSFR), translating into short (a few $100 \mathrm{Myr}$ ) stellar mass doubling times. The cumulative output of energy and momentum from stellar winds and $\mathrm{SNe}$ during such dominant phases of galaxy evolution will inevitably lead to large-scale gas thermalization and acceleration, with supershells protruding much beyond the galaxy itself. Extended nebular halos encompassing the still compact stellar component of high-SSFR proto-galactic systems may thus be ubiquitous in the early universe. The photometric structure of these galaxies could then closely resemble the right-hand panel of Fig. 12, comprising an HSB core within which the stellar component is confined and dominates and a much larger, nearly exponential, nebular LSB envelope. Despite cosmological dimming, the latter should be readily accessible to observations out to $z \gtrsim 2$, given that deep HST imaging and image stacking now permit studies of galaxies down to rest-frame surface brightnesses of $\gtrsim 26.5-28.5 \mathrm{mag} / \square^{\prime \prime}$ at those redshifts (e.g. Stockton et al. 2008; van Dokkum et al. 2010; Noeske et al. 2006). Morphological analogs to I Zw 18 may also exist among compact high-SSFR galaxies at intermediate redshift $(0.1 \lesssim z \lesssim 0.8)$, such as compact narrow emissionline galaxies (Koo et al. 1994; Guzmán et al. 1998), luminous compact blue galaxies (Guzmán et al. 2003; Puech et al. 2006) and green pea (GP) galaxies (Cardamone et al. 2009; Amorín et al. 2010; Izotov et al. 2011). The rest-frame $E W$ s of GPs can in some cases almost compete with those of nearby XBCDs (see e.g., Izotov et al. 2001b; Papaderos et al. 2006b).

Such considerations motivate a closer examination of observational biases that the spatial segregation between stellar and nebular emission may introduce into studies of distant, poorly resolved morphological analogs of I Zw 18. By appreciation of the differing spatial distribution of stellar and nebular emission, the discussion here goes beyond the framework of the state-ofart modeling studies that explore the impact of ne on the integral SED of SF galaxies in detail (e.g. Huchra 1977; Bergvall 1985; Salzer et al. 1989; Olofsson 1989; Krüger et al. 1995; Fioc \& Rocca-Volmerange 1997; Izotov et al. 1997a; Guseva et al. 2001; Moy et al. 2001; Anders et al. 2004; Zackrisson et al. 2001; Panuzzo et al. 2003; Zackrisson et al. 2008; Kotulla et al. 2009; Martín-Manjón et al. 2010; Schaerer \& de Barros 2009; Finlator et al. 2011; Ono et al. 2010, among others). The predictions from such zero-dimensional models are to be treated with some caution when compared with spatially resolved observables (e.g. radial $E W$ and color profiles) in order to place constraints on the formation history of galaxies. The minimum prerequisite for this approach to be valid is that nebular emission is cospatial with the local ionizing and non-ionizing stellar background, or, equivalently, that the ionizing Lyman continuum budget is reprocessed into nebular emission on the spot. That this idealized picture is not invariably valid and, actually, a strong spatial anticorrelation between emission line $E W \mathrm{~s}$ and stellar surface density may evolve over time both on small and large scales has been shown for several XBCDs (e.g. P98; P02; Papaderos et al. 1999; Izotov et al. 2001a; Guseva et al. 2001; Fricke et al. 2001). In these cases, subtracting synthetic ne SEDs from observed spectra, as done in P98, offers the only viable approach for isolating and age-dating the underlying stellar SED.

A discussion of aperture effects on the luminosity-weighted SEDs of distant morphological analogs to I $\mathrm{Zw} 18$ is beyond the scope of this study. Here, we only focus on potential photometric biases. A first one, already described in P02, arises from the fact that the nebular envelope may be taken as evidence for an evolved stellar disk, because of its exponentiality and reddish $(B-R$ and $V-R)$ rest frame colors. Automated surface photometry analyses of large extragalactic probes, if not including a robust discriminating criterion between stellar and nebular emission, could therefore be biased towards an enhanced frequency of galactic disks already in place at an early cosmic epoch. The arguably most convincing argument for this concern derives from I Zw 18 itself: if for one of the best studied galaxies in the local universe, it took three decades to realize that its exponential LSB luminosity envelope is not due to a stellar disk, but to ne, one may rightfully doubt that this would be immediately apparent in studies of its poorly resolved morphological analogs at high $z$.

Another potential bias comes from the fact that the superposition of two exponential profiles of differing $\mu_{0}$ and $\alpha-$ one representing the steeper star-dominated core and the other the shallower nebular envelope - can closely approximate a genuine Sérsic profile with a high shape parameter $(2 \leq \eta \lesssim 5)$, thereby mimicking the SBP of a massive spheroid. The best-fitting Sérsic exponent $\eta$ for such a composite SBP depends both on the properties of its constituent exponential profiles and on the limiting surface brightness $\mu_{\text {lim }}$ down to which Sérsic models are fitted. As an example, the best-fitting $\eta$ for I Zw 18 increases monotonically from 1.1 for $\mu_{\text {lim }}=23.5 \mathrm{mag} / \square^{\prime \prime}$ (at the surface brightness where ne takes over) to 2.2 for $\mu_{\text {lim }}=28 \mathrm{mag} / \square^{\prime \prime}$. This is illustrated in Fig. 13, where the observed SBP of I Zw 18 is compared with the superposition of two exponential components, approximating the core (profile labeled core) and the ne envelope (profile labeled env1). Their sum (core+env1) is then fitted by Sérsic models down to a progressively faint $\mu_{\mathrm{lim}}$. It can be seen from Fig. 14 that the best-fitting $\eta$ for the synthetic SBP is doubled when $\mu_{\text {lim }}$ decreases from 23.5 to $\geq 27.5$. The same 
P. Papaderos and G. Östlin: I Zw 18 as morphological paradigm for rapidly assembling high-z galaxies

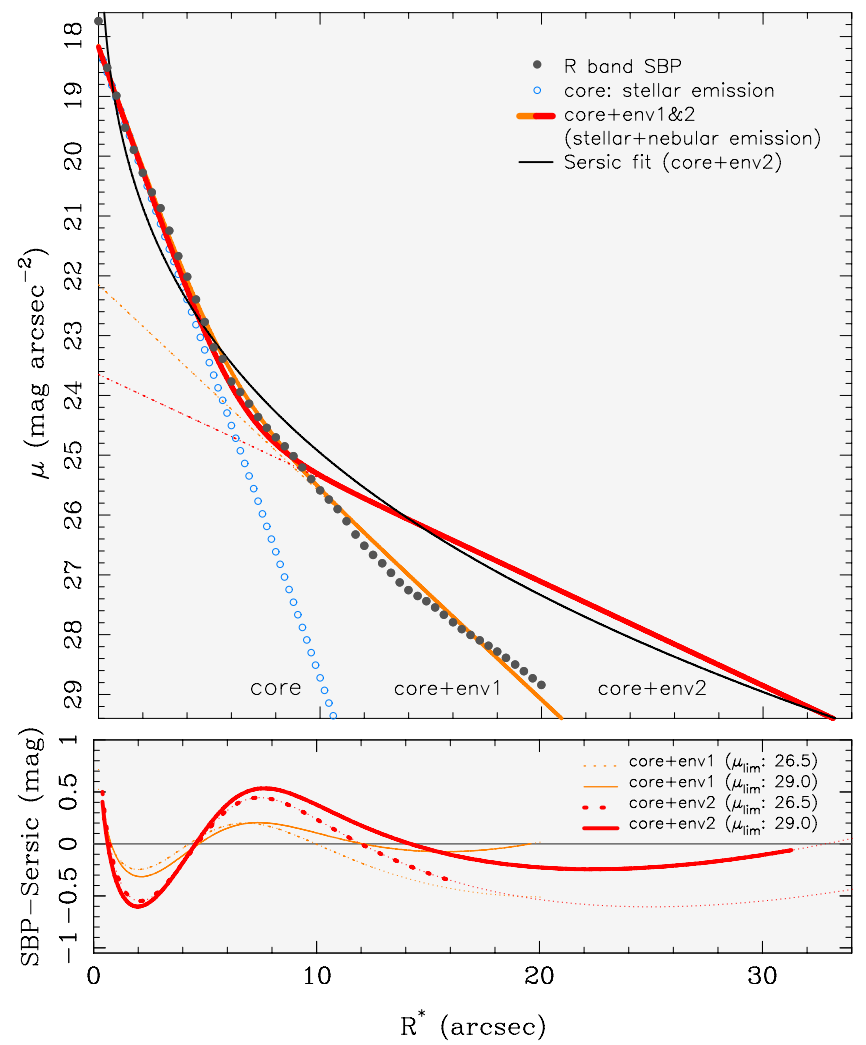

Fig. 13. Upper panel: comparison of the $R$ SBP of I Zw 18 (filled circles), with a synthetic SBP (labeled core+env1) that is due to the superposition of two exponential components of differing central surface brightness $\mu_{0}$ and exponential scale length $\alpha$. The first one, labeled core (open circles) approximates the stellar component that is confined to and dominates within the inner $\left(R^{\star} \leq 6^{\prime \prime}\right)$ HSB part of the observed SBP. The second component (env1; dotted line intersecting the abscisca at $\left.\mu \sim 22 \mathrm{mag} / \square^{\prime \prime}\right)$ is a linear fit to the nebular LSB envelope. A Sérsic model to the composite core+env1 SBP (orange solid-line curve) yields a Sérsic exponent $\eta \approx 2$, close to the best-fitting value of $\eta \approx 2.2$ for the observed SBP. The synthetic SBP labeled core+env2 (red solid-line curve) is computed by superposing on the core an exponential nebular envelope of equal luminosity but twice as large $\alpha$ as the observed envelope env1. A Sérsic fit to core+env2 (thin curve) yields an $\eta \approx 5$. Lower panel: residuals between the synthetic SBPs core+env1 (thin curve) and core+env2 (thick curve) and their Sérsic fits when the latter are computed down to a surface brightness level $\mu_{\lim }$ of 26.5 and $29 \mathrm{mag} / \square^{\prime \prime}$ (solid and dotted curves, respectively).

trend is recovered when Sérsic models are fitted directly to the observed SBP.

It is worth checking how the Sérsic $\eta$ vs. $\mu_{\text {eff }}$ relation may change in the case of an equally luminous but shallower nebular envelope. For this, we superpose on the core a component (env2) whose $\mu_{0}$ and $\alpha$ are by, respectively, 1.5 mag fainter and a factor of 2 larger than that in the observed component env1. The Sérsic fit to the core+env2 profile is included in Fig. 13. As is apparent in Fig. 14, in this case $\eta$ shows a steeper dependence on $\mu_{\text {lim }}$, increasing to $\sim 4$ already for $\mu_{\text {lim }} \simeq 26.5 \mathrm{mag} / \square^{\prime \prime}$ and leveling off to $\sim 5$ at fainter levels.

Consequently, Sérsic fits can easily lead to the misclassification of a compact high-SSFR galaxy as a massive elliptical and, quite counter-intuitively, deeper photometry exacerbates the problem. The residuals between SBP and model (lower panel of Fig. 13) are $\$ 0.5 \mathrm{mag}$ for core+env2 and just $\$ 0.2 \mathrm{mag}$ for core+env1, i.e. on the order of $1 \sigma$ uncertainties in SBPs of intermediate-to-high $z$ galaxies (cf. e.g. Noeske et al. 2006), i.e.

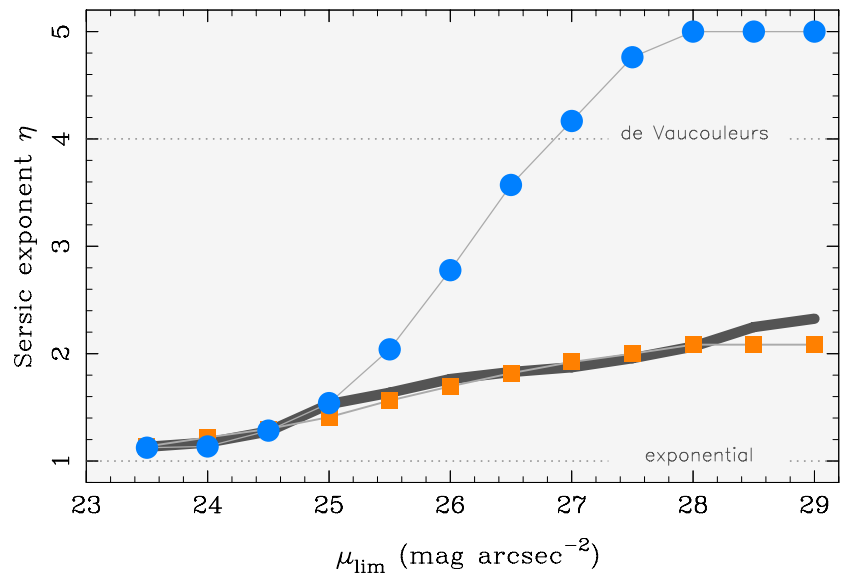

Fig. 14. Best-fitting Sérsic exponent $\eta$ vs. $\mu_{\text {lim }}$ for the SBPs in Fig. 13. The thick-gray curve and the filled squares show, respectively, the variation in $\eta$ for the observed $R$ band SBP of I Zw 18 and the twocomponent approximation to it, labeled core+env1. The variation in $\eta$ with $\mu_{\text {eff }}$ for the synthetic SBP core+env2 is shown with filled circles.

small enough to go undetected. In practice, even when PSF convolution effects are fully accounted for, the pseudo-Sérsic profile of a compact diskless high-SSFR galaxy is barely distinguishable from the Sérsic profile of a massive galaxy spheroid.

Evidently, since extended ne can drastically affect an SBP as a whole, it also affects virtually all secondary photometric parameters that are derivable from it, e.g., the effective radius and Gini coefficient, and the various light concentration indices commonly used in galaxy quantitative morphology studies.

Moreover, the ne luminosity fraction in I Zw $18(\geq 1 / 3)$, if typical of its higher- $z$ analogs, translates into a systematic error of $\geq 0.4$ mag in galaxy scaling relations involving total magnitudes. These range from the Tully-Fisher relation to all relations comparing luminosity with e.g. metallicity, diameter, mean surface brightness and velocity dispersion. Errors in galaxy luminosity propagate, potentially amplified, in stellar mass determinations using theoretical mass-to-light ratios or SED fitting. An investigation of this issue was recently presented by Izotov et al. (2011): these authors show that masses computed from spectral fits to the SED continuum of high-SSFR galaxies at intermediate $z$ can be overestimated by a factor of up to $\sim 4$. This is not primarily due to the luminosity contribution but more to the red spectral slope of the nebular continuum (see e.g. Krüger et al. 1995) that drives SED fitting towards solutions invoking much too high a luminosity fraction from old stars. In galaxy assembly studies covering a wide range in $z$, downsizing effects may further complicate the aforementioned biases, since ne is expected to affect galaxy populations of different mass over different timescales.

We next turn to the color contrast $\delta_{\text {ce }}$ between the HSB core and the nebular LSB envelope. This quantity can be determined easily from SBPs, or within concentric apertures, and provides a handy proxy to radial color gradients in galaxies, thus a first guess at classification. As we show below, for certain $z$ intervals, $\delta_{\text {ce }}$ offers a powerful diagnostic for identifying high-SSFR galaxies with morphological properties analogous to those of I Zw 18. Within other $z$ intervals, however, and depending on the colors considered, a superficial interpretation of $\delta_{\text {ce }}$ can further aggravate the above discussed galaxy misclassification biases.

In computing $\delta_{\mathrm{ce}}$ and its variation with $z$, we approximated the spectrum of I Zw 18 with synthetic stellar + ne SEDs from Pegase 2, referring to SFH2 and a metallicity $Z=0.0004$. In 
these models, the properties of the HSB core $\left(R^{\star} \leq 6^{\prime \prime}\right)$ are reproduced well by a synthetic SED for an age $t=100 \mathrm{Myr}$. This yields colors of $V-R=0.2, V-I=-0.15, R-I=-0.37 \mathrm{mag}$, and an $E W(\mathrm{H} \alpha)$ of $670 \AA$, in good agreement with the observed values (Izotov et al. 2001a; Vílchez \& Iglesias-Páramo 1998, P02). As for the envelope $\left(6^{\prime \prime} \leq R^{\star} \lesssim 20^{\prime \prime}\right)$, we adopted the SED from the same model for $t=0$, i.e. a purely ne spectrum. Its colors $(B-V=0.28, V-R=0.47, B-R=0.7, V-I=-0.65$, and $R-I=-1.1 \mathrm{mag}$ ) provide a good match to the data as well.

The variation in the $B-J, V-K, V-R, V-I$, and $R-I$ colors of the core and in the envelope as a function of $z$ is shown in the upper two panels of Fig. 15. It can be seen that the envelope (middle panel) shows particularly strong color variations, because different strong emission lines shift into the transmittance window of various filters, depending on $z$. As is already evident from Sect. 3.2, a local analog to I Zw 18 is easily identified by its blue nebular envelope and large $(0.8 \mathrm{mag}) \delta_{\text {ce }}$ (lower panel) both with respect to $V-I$ and $R-I$. This is also the case for the redshift range $0.15 \lesssim z \lesssim 0.3$ where the envelope appears much redder than the core in $V-I$ but bluer in $B-V$. Other distinct peaks in $\left\|\delta_{\text {ce }}\right\|$ with respect to various optical or optical-NIR colors are apparent for, e.g., $z \approx 0.42,0.55$, and 0.9 . A comparison of the upper and lower panel of Fig. 15 shows that the $\delta_{\text {ce }}$ exhibits much larger variations $\left(\left\|\delta_{\text {ce }}\right\| \simeq 1.6 \mathrm{mag}\right)$ than the HSB core; i.e., it is a far more sensitive indicator of extended ne than integral (luminosity-weighted) colors that are primarily driven by the core. For example, at $z \approx 0.27$, the $V-I$ and $B-V$ colors of the HSB core are equal to within $\leq 0.3 \mathrm{mag}$, whereas their $\delta_{\text {ce }}$ differs by up to $\approx 1.3$ mag.

It is worth pointing out that, for certain redshift windows and color indices, the $\delta_{\text {ce }}$ may point towards diametrically different views on the nature of an I Zw 18 analog. For instance, at $0.15 \lessgtr z \lesssim 0.3$, the large $V-I$ core-to-envelope color contrast $\left(\delta_{\mathrm{ce}} \sim 0.8 \mathrm{mag}\right)$ and the moderately blue colors of the core $(0.5 \mathrm{mag})$ superficially suggest an old disk hosting nuclear SF activity. The opposite conclusion could be drawn from the $\delta_{\text {ce }}$ in $B-V(\approx 0.5 \mathrm{mag})$, which may be taken as evidence of a very young stellar disk encompassing a slightly older core, in line with the inside-out galaxy growth interpretation. In either case, the exponential envelope, usually interpreted as a stellar disk, would nicely augment the erroneous conclusion. Several similar cases for various color combinations and redshifts can be read off Fig. 15. Important is also that for other $z$ 's (e.g. $0.1 \lesssim z \lesssim 0.15,0.3,0.75,1.05)$, the $\delta_{\text {ce }}$ vanishes to $\lesssim 0.2 \mathrm{mag}$ for some colors, with little discriminating power between stellar and nebular emission, and superficially suggesting a nearly uniform stellar age.

With regard to all concerns above, one might counterargue that photometric $k$ corrections would rectify SBPs and color profiles, and eliminate pitfalls in the interpretation of $\delta_{\text {ce }}$. However, state-of-art $k$ corrections, mostly tailored to stellar SEDs and applied to a morphological analog of I Zw 18 as a whole, regardless of its physically distinct radial zones, would most probably aggravate the problem in a barely predictable manner.

In summary, the case of I ZW 18 stands as a warning benchmark to studies of high-SSFR galaxies near and far. It reminds us that nebular emission in SF galaxies is not always cospatial with the underlying local ionizing and non-ionizing stellar background, and it does not have to scale with its surface density. Quite the contrary, in galaxies with high SSFR (i.e. forming young galaxies and/or systems rapidly assembling their stellar mass during dominant phases of their evolution), nebular emission is plausibly expected to extend far beyond the galaxy itself and to contribute significantly to its total luminosity. This,

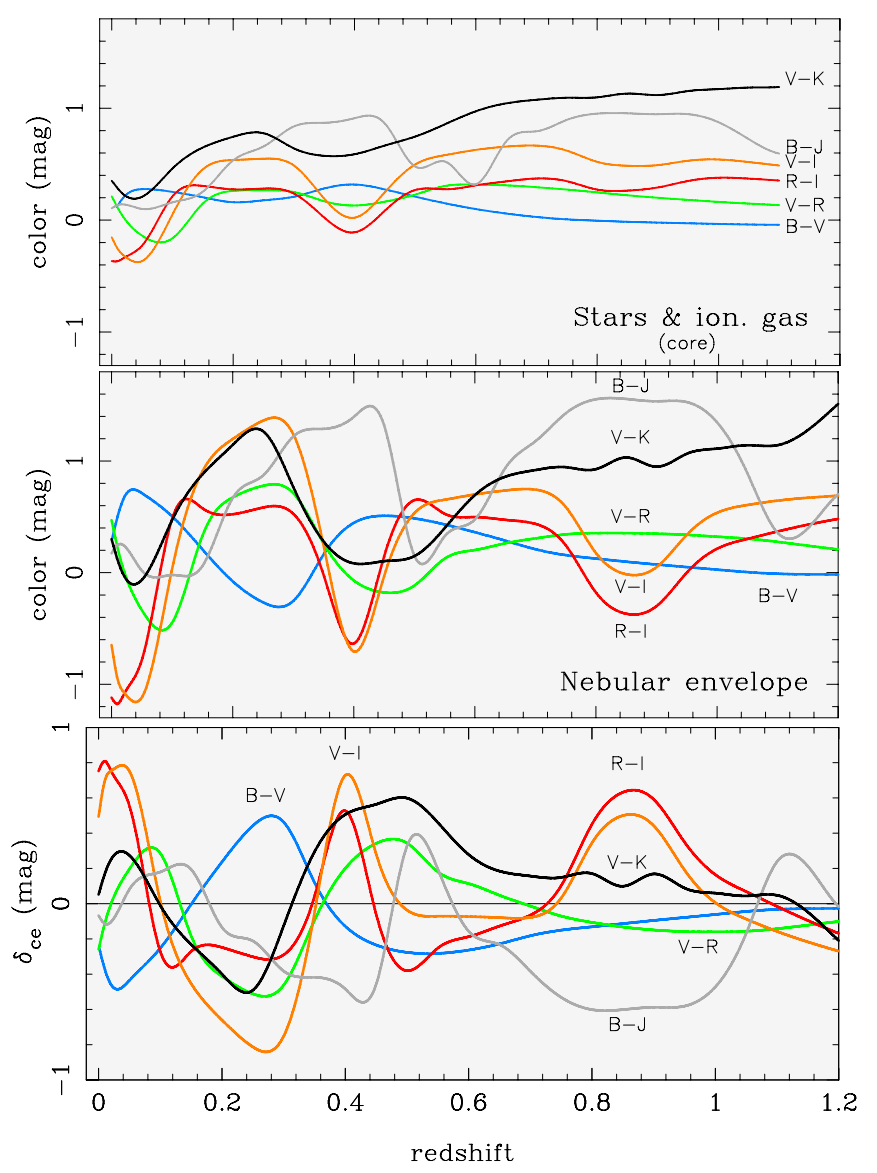

Fig. 15. Variation in the observed color as a function of redshift $z$ for a template galaxy with the properties of I Zw 18. The model consists of an HSB core within which stellar emission is confined and dominates (upper panel), and a larger nebular LSB envelope (middle panel). The lower panel shows the dependence of the color contrast $\delta_{\text {ce }}$ between the core and the envelope as a function of redshift.

in connection with the empirical evidence that nebular emission forms an exponential envelope, conspires in potentially important observational biases in studies of moderately resolved morphological analogs to I $\mathrm{Zw} 18$, which are likely ubiquitous in the early universe.

Interesting in this context is also the detection of exponential Ly $\alpha$ halos around low (Hayes et al. 2007) and high- $z$ SF galaxies (Steidel et al. 2011), resulting in SBPs strikingly similar to those of I Zw 18.

In principle, any strong thermal or non-thermal central source of energy and momentum could generate a large nebular envelope and a pseudo bulge-disk luminosity profile. The foregoing discussion is therefore relevant to a wider range of topics, such as the co-evolution of AGNs with their host galaxies or the properties of ionized halos around powerful radio galaxies (e.g., Villar-Martín et al. 2003, and references therein).

The prospect of spatially resolved studies of galaxy formation in the faraway universe with next-generation observing facilities calls for theoretical guidance with the properties and time evolution of nebular halos around protogalactic systems. Some questions (see also P02) to computational astrophysics include i) in which way are the photometric properties of the nebular envelope (e.g. its $\mu_{0}$ and $\alpha$ ) and their temporal evolution related to the specifics of energy production (e.g. the SFH and the ionizing SED), the initial geometry, and kinematics of the protogalactic gas reservoir and the physical conditions in the 
intergalactic medium (e.g. external pressure, ambient ionizing field from multiple mutually "illuminating" protogalactic units)? More specifically; ii) does (for a given set of environmental conditions) the core-envelope radial intensity pattern scale in a homologous manner; i.e., is the $\alpha$ of the nebular envelope invariant? If not; iii) what does the core-to-envelope luminosity and normalized $R_{\mathrm{SF}} / \alpha$ ratio tell us about, e.g., the recent $\mathrm{SFH}$ and current SSFR? Additionally; iv) how is the photometric, chemical and kinematical evolution of the nebular envelope related to the escape probability of Ly continuum and Ly $\alpha$ photons?

\section{Summary and conclusions}

We used the entire set of archival HST ACS broad band imaging data for I $\mathrm{Zw} 18$ and its fainter component I $\mathrm{Zw} 18 \mathrm{C}$ to study the photometric structure of this nearby $(19 \mathrm{Mpc})$ dwarf galaxy pair to unprecedently faint surface brightness levels $(\mu \gtrsim$ $\left.29 \mathrm{mag} / \square^{\prime \prime}\right)$. The main results from this study may be summarized as follows:

i) Radial color profiles reveal very blue and practically constant colors $(0 \pm 0.05 \mathrm{mag})$ for $\mathrm{I} \mathrm{Zw} 18 \mathrm{C}$ down to $\mu$ $27.6 \mathrm{mag} / \square^{\prime \prime}$, and a previously undisclosed, slightly redder $(V-I=0.2 \pm 0.08 \mathrm{mag}, R-I=0.16 \pm 0.09 \mathrm{mag})$ stellar component in its extreme periphery (27.6-29 mag/ $\left.\square^{\prime \prime}\right)$. We verified that these blue colors do not merely reflect the luminosity-weighted average of blue young stellar clusters with a faint red stellar background but that they are characteristic for the unresolved host galaxy of I Zw $18 \mathrm{C}$. We argued that the buildup of the photometrically dominant stellar component of I Zw $18 \mathrm{C}$ has occurred in a largely sequential mode, through an SF process that likely started $\tau \sim 100 \mathrm{Myr}$ ago at the redder northwestern tip of the galaxy and propagated with a mean velocity of $\sim 20 \mathrm{~km} \mathrm{~s}^{-1}$ to its bluer southeastern tip.

The photometric properties of the extreme periphery of I $\mathrm{Zw} 18 \mathrm{C}$ are entirely consistent with this formation scenario, if the effect of stellar diffusion is taken into account. Radial migration of newly forming stars with a mean velocity of $\approx 4 \mathrm{~km} \mathrm{~s}^{-1}$ over $\sim \tau$, and the associated stellar mass filtering effect described in Papaderos et al. (2002, hereafter P02), can naturally account for the slightly redder colors, absence of nebular emission (ne) and topological properties of the stellar outskirts of I Zw $18 \mathrm{C}$. A faint ancient stellar substrate cannot be ruled out, even though our analysis does not lend observational support to its presence.

ii) In I Zw 18 (i.e. the main body) severe contamination of broad band colors by ne prevents a conclusive age dating of the stellar component almost everywhere. Therefore, even though our combined images are the deepest presently available, we cannot improve on the age-dating analysis by $\mathrm{P} 02$ who have 2D subtracted strong nebular emission lines from broad band HST WFPC2 images to isolate and age-date the residual underlying stellar background. However, the colors of the reddest quartile of region $\omega$, a region at the southeastern tip of I $\mathrm{Zw} 18$ with comparatively weak ne, were determined to be in good agreement with those previously inferred by P02. Assuming these colors are representative of the host galaxy of I $\mathrm{Zw} 18$, and on the basis of a continuous or exponentially decreasing SFR model, it follows that I Zw 18 has formed most of its stellar mass at a late cosmic epoch. This, together with our conclusions under i), supports the view that both I Zw 18 and I Zw $18 \mathrm{C}$ are cosmologically young objects that have undergone a nearly synchronous evolution. iii) We showed that $\sim 20 \%$ of the isophotal area of I $\mathrm{Zw} 18$ at $25 \mathrm{mag} / \square^{\prime \prime}$ is severely affected by ne, thus inaccessible to age-dating studies via broad band colors and colormagnitude diagrams (CMDs). The local impact of ne manifests itself in, e.g., a combination of red ( $\sim 0.5 \mathrm{mag}) V-R$ with extremely blue $(-0.4 \ldots-0.8 \mathrm{mag}) V-I$ and $R-I$ colors. Nebular emission shows considerable sub-structure, with numerous clumps and overlapping shells, and little spatial correlation with the local stellar background. It thus cannot be treated as a uniform foreground emitting layer and accurately subtracted out using standard point source photometry algorithms. This likely results in substantial random and systematic errors that might not be fully accounted for by the standard CMD error budget. Further potential sources of systematic uncertainties stem from spatial displacements $(50-100 \mathrm{pc})$ between the intensity maxima of the [OIII] $\lambda \lambda 4959,5007$ and $\mathrm{H} \alpha$ emission lines along ionized gas shells. These may differentially affect the local background determination in different filters, causing an artificial reddening of CMD point sources in the interior of nebular shells (and vice versa).

iv) Based on the extraordinarily deep combined HST ACS images, we were able to study the exponential LSB envelope of I $\mathrm{Zw} 18$ out to its extreme periphery using both SBPs and color maps. These reveal uniform colors of $V-R \approx 0.55 \mathrm{mag}$, $V-I \approx-1 \mathrm{mag}$, and $R-I \approx-1.4 \mathrm{mag}$ all over the LSB component of I $\mathrm{ZW} \mathrm{18}$, corroborating the previous conclusion by $\mathrm{P} 02$ that this luminosity envelope is not due to a stellar disk, but to extended ne. Specifically, our analysis indicates that ne dominates the line-of-sight intensity beyond three effective radii (or, equivalently, for $\mu \geq 23.5 \mathrm{mag} / \square^{\prime \prime}$ ) and extends as far out as $\sim 16$ stellar exponential scale lengths. The overall picture emerging from our analysis is therefore that I $\mathrm{Zw} 18$ is a cosmologically young object that consists of a compact, HSB core, within which stellar emission is confined and dominates, and a much larger nebular LSB envelope.

v) We argued that the morphological properties of I $\mathrm{Zw} 18$, while unique among nearby SF dwarf galaxies, are probably typical of distant young galaxies in dominant phases of their evolution, during which they assemble their stellar component at high SSFRs. The prodigious energetic output during such phases of rapid stellar mass growth is expected to result into a large-scale gas ionization and acceleration, with ne protruding much beyond the still compact stellar galaxy host. These systems could thus bear strong morphological resemblance to $\mathrm{I} \mathrm{Zw} 18$, comprising a compact core that is dominated by stellar emission and a much larger exponential nebular envelope. A question of considerable interest is, therefore, how the nebular envelope could affect photometric studies of moderately resolved morphological analogs of I Zw 18 at higher $z$.

A potential bias, already discussed in $\mathrm{P} 02$, arises from the nebular envelope mimicking an evolved stellar disk by its exponentiality and red $B-R$ color. Here, by using I Zw 18 as a template, we extended previous considerations.

v.1 We pointed out that the superposition of two exponential components of differing central surface brightness and scale length, approximating the core and the envelope of a distant I ZW 18 analog, may be barely distinguishable from a genuine Sérsic profile with an exponent $2 \lesssim \eta \lesssim 5$. Therefore, Sérsic models offer, in the specific context, a poor diagnostic for disentangling compact high-SSFR protogalaxies from massive galaxy spheroids. 
v.2 Nebular emission contributes at least one third of the total luminosity of I Zw 18 (P02 and this study). This luminosity fraction, if typical of its higher- $z$ analogs, translates into a systematic error of $\gtrsim 0.4 \mathrm{mag}$ in all galaxy scaling relations involving luminosities (e.g., the Tully-Fisher relation, and relations between luminosity and metallicity, diameter, velocity dispersion). Evidently, errors in total luminosities propagate into errors in galaxy mass determinations via theoretical mass-to-light ratios or SED fitting techniques. Moreover, since extended ne can drastically affect a galaxy SBP as a whole, it also affects practically all secondary photometric quantities that are derivable from it (e.g. the effective radius or various light concentration indices used for quantitative galaxy morphology studies).

v.3 We investigated the variation in the color contrast $\delta_{\text {ce }}$ between the star-dominated core and the surrounding nebular envelope as a function of $z$. This task is motivated by the fact that $\delta_{\text {ce }}$ provides a handy proxy to radial color gradients in galaxies and a valuable galaxy classification tool. We show that, for certain $z$ intervals, this quantity offers a powerful diagnostic for identifying moderately resolved I Zw 18 analogs. Within other $z$ intervals, however, and depending on the color indices considered, a superficial interpretation of $\delta_{\text {ce }}$ can further enhance galaxy misclassification biases stemming from SBP fitting (cf. v.1) and potentially affect our understanding of galaxy assembly over time. State-of-art $k$ corrections applied to distant morphological analogs to I Zw 18 as a whole, i.e. regardless of their physically distinct radial zones, may aggravate observational and interpretation biases in a barely predictable manner.

In the era of spatially resolved studies of galaxy formation in the early universe with next-generation observing facilities, a better theoretical understanding of the rise and fall of nebular galaxy halos over cosmic time appears to be crucial. In this respect, we have formulated some questions for computational astrophysics.

Acknowledgements. Polychronis Papaderos is supported by a Ciencia 2008 contract, funded by FCT/MCTES (Portugal) and POPH/FSE (EC). He also acknowledges support by the Wenner-Gren Foundation. Göran Östlin acknowledges support from the Swedish Research council (VR) and the Swedish National Space Board (SNSB). He is a Royal Swedish Academy of Sciences research fellow, supported by a grant from the Knut and Alice Wallenberg foundation. A careful reading and constructive report by the referee is very much appreciated. This research made use of data acquired with the European Southern Observatory telescopes, and obtained from the ESO/ST-ECF Science Archive Facility and of the NASA/IPAC Extragalactic Database (NED), which is operated by the Jet Propulsion Laboratory, CALTECH, under contract with the National Aeronautic and Space Administration.

\section{References}

Aloisi, A., Tosi, M., \& Greggio, L. 1999, AJ, 118, 302

Aloisi, A., Savaglio, S., Heckman, T. M., et al. 2003, ApJ, 595, 760

Amorín, R., Alfonso, J., Aguerri, L., Muñoz-Tuñón, C., \& Cairós, L. M. 2009, A\&A, 501, 75

Amorín, R., Pérez-Montero, E., \& Vílchez, J. M. 2010, ApJ, 715, L128

Anders, P., de Grijs, R., Fritze-v. Alvensleben, U., \& Bissantz, N. 2004, MNRAS, 347, 17

Beckwith, S. V. W., Stiavelli, M., Koekemoer, A. M., et al. 2006, AJ, 132, 1729

Bender, R., \& Möllenhoff, C. 1987, A\&A, 177, 71

Bergvall, N. 1985, A\&A, 146, 269

Bergvall, N., \& Östlin, G. 2002, A\&A, 390, 891

Bergvall, N., Zackrisson, E., \& Caldwell, B. 2010, MNRAS, 405, 2697

Binggeli, B., \& Cameron, L. M. 1991, A\&A, 252, 27

Binggeli, B., \& Cameron, L. M. 1993, A\&AS, 98, 297

Bomans, D. J. 2002, in Dwarf Galaxies and their Environment, ed. K. S. de Boer, R.-J. Dettmar, \& U. Klein (Shaker Verlag), 145

Cairós, L. M., Vílchez, J. M., Gónzalez-Pérez, J. N., Iglesias-Páramo, J., \& Caon, N. 2001, ApJS, 133, 321
Cairós, L. M., Caon, N., García Lorenzo, B., Vílchez, J. M., \& Muñoz-Tuñón, C. 2002, ApJ, 577, 164

Cairós, L. M., Caon, N., Papaderos, P., et al. 2009, ApJ, 707, 1676

Cannon, J. M., Skillman, E. D., Garnett, D. R., \& Dufour, R. J. 2002, ApJ, 565, 931

Cardamone, C., Schawinski, K., Sarzi, M., et al. 2009, MNRAS, 399, 1191

Cerviño, M., \& Mas-Hesse, J. M. 1994, A\&A, 284, 749

Crone, M. M., Schulte-Ladbeck, R. E., Greggio, L., \& Hopp, U. 2002, ApJ, 567, 258

Davidson, K., Kinman, T. D., \& Friedman, S. D. 1989, AJ, 97, 1591

Dufour, R. J., \& Hester, J. J. 1990, ApJ, 350, 149

Dufour, R. J., Esteban, C., \& Castañeda, H. O. 1996a, ApJ, 471, L87

Dufour, R. J., Garnett, D. R., Skillman, E. D., \& Shields, G. A. 1996b, in From Stars To Galaxies, ed. C. Leitherer, U. Fritze-v. Alvensleben, \& J. Huchra, ASP Conf. Ser., 98, 358

Ferguson, A. M. N., Wyse, R. F. G., Gallagher, J. S., \& Hunter, D. A. 1996, AJ, 111,2265

Finlator, K., Oppenheimer, B. D., \& Davé, R. 2011, MNRAS, 410, 1703

Fioc, M., \& Rocca-Volmerange, B. 1997, A\&A, 326, 950

Fiorentino, G., Contreras Ramos, R., Clementini, G., et al. 2010, ApJ, 711, 808 French, H. B. 1980, ApJ, 240, 41

Fricke, K. J., Izotov, Y. I., Papaderos, P., Guseva, N. G., \& Thuan, T. X. 2001, AJ, 121, 169

Gallagher, J. S., Bushouse, H., \& Hunter, D. A. 1989, AJ, 97, 700

Gil de Paz, A., \& Madore, B. F. 2005, ApJS, 156, 345

Gil de Paz, A., Madore, B. F., \& Pevunova, O. 2003, ApJS, 147, 29

Guseva, N. G., Izotov, Y. I., Papaderos, P., et al. 2001, A\&A, 378, 756

Guseva, N. G., Papaderos, P., Izotov, Y. I., Noeske, K. G., \& Fricke, K. J. 2004, A\&A, 421, 519

Guseva, N. G., Papaderos, P., Meyer, H. T., Izotov, Y. I., \& Fricke, K. J. 2009, A\&A, 505, 63

Guzmán, R., Jangren, A., Koo, D. C., Bershady, M. A., \& Simard, L. 1998, ApJ, 495, L13

Guzmán, R., Östlin, G., Kunth, D., et al. 2003, ApJ, 586, L45

Hayes, M., Östlin, G., Hakim, A., et al. 2007, MNRAS, 382, 1465

Huchra, J. P. 1977, ApJ, 217, 928

Hunt, L. K., Thuan, T. X., \& Izotov, Y. I. 2003, ApJ, 588, 281

Hunter, D. A., \& Gallagher, J. S. 1985, ApJS, 58, 533

Hunter, D. A., \& Gallagher, J. S. 1990, ApJ, 362, 480

Hunter, D. A., \& Gallagher, J. S. 1992, ApJ, 391, L9

Hunter, D. A., \& Thronson, H. A., Jr. 1995, ApJ, 452, 238

Izotov, Y. I., \& Thuan, T. X. 1998a, ApJ, 497, 227

Izotov, Y. I., \& Thuan, T. X. 1998b, ApJ, 500, 188

Izotov, Y. I., \& Thuan, T. X. 1999, ApJ, 511, 639

Izotov, Y. I., \& Thuan, T. X. 2004, ApJ, 616, 768

Izotov, Y. I., \& Thuan, T. X. 2007, ApJ, 665, 1115

Izotov, Y. I., Lipovetsky, V. A., et al. 1997a, ApJ, 476, 698

Izotov, Y. I., Foltz, C. B., Green, R. F., Guseva, N. G., \& Thuan, T. X. 1997b, ApJ, 487, L37

Izotov, Y. I., Papaderos, P., Thuan, T. X., et al. 1999a, unpublished [arXiv: astro-ph/9907082]

Izotov, Y. I., Chaffee, F. H., Foltz, C. B., et al. 1999b, ApJ, 527, 757

Izotov, Y. I., Chaffee, F. H., Foltz, C. B., et al. 2001a, ApJ, 560, 222

Izotov, Y. I., Chaffee, F. H., \& Schaerer, D. 2001b, A\&A, 378, L45

Izotov, Y. I., Thuan, T. X., \& Guseva, N. G. 2005, ApJ, 415, 87

Izotov, Y. I., Schaerer, D., Blecha, A., et al. 2006, A\&A, 459, 71

Izotov, Y. I., Guseva, N. G., Fricke, K. J., \& Papaderos, P. 2009, A\&A, 503, 61

Izotov, Y. I., Guseva, N. G., \& Thuan, T. X. 2011, ApJ, 727, 161

Jamet, L., Cerviño, M., Luridiana, V., Pérez, E., \& Yakobchuk, T. 2010, A\&A 509, A10

Jee, M. J., Blakeslee, J. P., Sirianni, M., et al. 2007, PASP, 119, 1403

Kinman, T. D., \& Davidson, K. 1981, ApJ, 243, 127

Knollmann, S. R. 2004, Diploma Thesis, University of Göttingen

Koo, D. C., Bershady, M. A., Wirth, G. D., Stanford, S. A., \& Majewski, S. R. 1994, ApJ, 427, L9

Koopmann, R. A., \& Kenney, J. D. P. 2006, ApJS, 162, 97

Kotulla, R., Fritze, U., Weilbacher, P., \& Anders, P. 2009, MNRAS, 396, 462

Krüger, H., Fritze-v. Alvensleben, U., \& Loose, H.-H. 1995, A\&A, 303, 41

Kunth, D., \& Östlin, G. 2000, A\&ARv, 10, 1 (KÖ00)

Kunth, D., Lequeux, J., Sargent, W. L. W., \& Viallefond, F. 1994, A\&A, 282, 709

Lecavelier des Etangs, A., Désert, J.-M., Kunth, D., et al. 2004, A\&A, 413, 131

Legrand, F. 2000, A\&A, 354, 504

Legrand, F., Kunth, D., Roy, J.-R., Mas-Hesse, J. M., \& Walsh, J. R. 1997, A\&A, 326, L17

Legrand, F., Tenorio-Tagle, G., Silich, S., Kunth, D., \& Cerviño, M. 2001, ApJ, 560,630

Lee, J. C., Kennicutt, R. C., Funes, S. J., et al. 2007, ApJ, 671, L113 
P. Papaderos and G. Östlin: I Zw 18 as morphological paradigm for rapidly assembling high- $z$ galaxies

Leitherer, C., Schaerer, D., Goldader, J. D., et al. 1999, ApJS, 123, 3

Lequeux, J., Rayo, J. F., Serrano, A., Peimbert, M., \& Torres-Peimbert, S. 1979, A\&A, 80, 155

Leroy, A., Cannon, J., Walter, F., Bolatto, A., \& Weiss, A. 2007, ApJ, 663, 990

Loose, H.-H., \& Thuan, T. X. 1986, in Star Forming Dwarf Galaxies and Related Objects, ed. D. Kunth, T. X. Thuan, \& J. Tran Thanh Van (Éditions Frontières), 73 (LT86)

Marlowe, A. T., Heckman, T. M., Wyse, R. F. G., \& Schommer, R. 1995, ApJ, 438, 563

Martin, C. L. 1996, ApJ, 465, 680

Martin, C. L. 1998, ApJ, 506, 222

Martín-Manjón, M. L., García-Vargas, M. L., Mollá, M., \& Díaz, A. I. 2010, MNRAS, 403, 2012

Mateo, M. 1998, ARA\&A, 36, 435

Matteucci, F., \& Tosi, M. 1985, MNRAS, 217, 39

Mas-Hesse, J. M., \& Kunth, D. 1999, A\&A, 349, 765

McCray, R., \& Kafatos, M. 1987, ApJ, 317, 190

Meurer, G. R., Freeman, K. C., Dopita, M. A., \& Cacciari, C. 1992, AJ, 103, 60

Momany, Y., Held, E. V., Saviane, L. R., et al. 2005, A\&A, 439, 111

Monreal-Ibero, A., Vílchez, J. M., Walsh, J. R., \& Muñoz-Tuñón, C. 2010, A\&A, 517, A27

Moustakas, J., \& Kennicutt, R. C. 2006, ApJS, 164, 81

Moy, E., Rocca-Volmerange, B., \& Fioc, M. 2001, A\&A, 365, 347

Noeske, K. G., Papaderos, P., Cairós, L. M., \& Fricke, K. J. 2003, A\&A, 410, 481

Noeske, K. G., Koo, D. C., Phillips, A. C., et al. 2006, ApJ, 640, L143

Olofsson, K. 1989, A\&A, 80, 317

Ono, Y., Ouchi, M., Shimasaku, K., et al. 2010, ApJ, 724, 1524

Östlin, G. 2000, ApJ, 535, 99

Östlin, G., \& Mouhcine, M. 2005, A\&A, 433, 797

Östlin, G., Bergvall, N., \& Rönnback, J. 1996, in The Interplay Between Massive Star Formation, the ISM and Galaxy Formation, ed. D. Kunth, B. Guiderdoni, M. Heydari-Malayeri \& T. X. Thuan (Gif-sur-Yvette: Éditions Frontières), 605

Östlin, G., Zackrisson, E., Bergvall, N., \& Rönnback, J. 2003, A\&A, 408, 887

Pagel, B. E. J., Simonson, E. A., Terlevich, R. J., \& Edmunds, M. G. 1992, MNRAS, 255, 325

Panuzzo, P., Bressan, A., Granato, G. L., Silva, L., \& Danese, L. 2003, A\&A, 409, 99

Papaderos, P., \& Fricke, K. J. 1998, in Highlights in X-ray Astronomy, ed. B. Aschenbach, \& M. J. Freyberg, MPE Report, 272, 193

Papaderos, P., Loose, H.-H., Thuan, T. X., \& Fricke, K. J. 1996a, A\&AS, 120, 207 (P96a)

Papaderos, P., Loose, H.-H., Fricke, K. J., \& Thuan, T. X. 1996b, A\&A, 314, 59 (P96b)

Papaderos, P., Izotov, Y. I., Fricke, K. J., Thuan, T. X., \& Guseva, N. G. 1998, A\&A, 338, 43 (P98)

Papaderos, P., Fricke, K. J., Thuan, T. X., Izotov, Y. I., \& Nicklas, H. 1999, A\&A, 352, L57

Papaderos, P., Izotov, Y. I., Noeske, K. G., Thuan, T. X., \& Fricke, K. J. 2001, in Dwarf Galaxies and their Environment, ed. K. S. de Boer, R.-J. Dettmar \& U. Klein (Shaker Verlag), 111

Papaderos, P., Izotov, Y. I., Thuan, T. X., et al. 2002, A\&A, 393, 461 (P02)

Papaderos, P., Izotov, Y. I., Guseva, N. G., Thuan, T. X., \& Fricke, K. J. 2006a, A\&A, 454, 119

Papaderos, P., Guseva, N. G., Izotov, Y. I., et al. 2006b, A\&A, 457, 45

Papaderos, P., Guseva, N. G., Izotov, Y. I., \& Fricke, K. J. 2008, A\&A, 491, 113
Patterson, R. J., \& Thuan, T. X. 1996, ApJS, 107, 103

Peng, C. Y., Ho, L. C., Impey, C. D., \& Rix, H.-W. 2002, AJ, 124, 266

Péquignot, D. 2008, A\&A, 478, 371

Petrosian, A. R., Boulesteix, J., Comte, G., Kunth, D., \& LeCoarer, E. 1997, A\&A, 318, 390

Pflamm-Altenburg, J., Weidner, C., \& Kroupa, P. 2009, MNRAS, 395, 394

Puech, M., Hammer, F., Flores, H., Östlin, G., \& Marquart, T. 2006, A\&A, 455, 119

Pustilnik, S. A., Kniazev, A. Y., \& Pramskij, A. G. 2005, A\&A, 443, 91

Recchi, S., Matteucci, F., D’Ercole, A., \& Tosi, M. 2004, A\&A, 426, 37

Reines, A. E., Johnson, K. E., \& Hunt, L. K. 2008, AJ, 136, 1415

Relaño, M., Monreal-Ibero, A., Vílchez, J. M., \& Kennicutt, R. C. 2010 MNRAS, 402, 1635

Reverte, D., Vílchez, J. M., Hernández-Fernández, J. D., \& Iglesias-Páramo, J. 2007, AJ, 133, 705

Rönnback, J., \& Bergvall, N. 1994, A\&AS, 108, 193

Roy, J.-R., \& Kunth, D. 1995, A\&A, 294, 432

Salzer, J. J., MacAlpine, G. M., \& Boroson, T. A. 1989, ApJS, 70, 447

Sánchez Almeida, J., Muñoz-Tuñón, C., Amorín, R., et al. 2008, ApJ, 685, 194

Sargent, W. L. W., \& Searle, L. 1970, ApJ, 162, L155

Searle, L., \& Sargent, W. L. W. 1972, ApJ, 173, 25

Schaerer, D., \& de Barros, S. 2009, A\&A, 502, 423

Simard, L. 1998, in Astronomical Data Analysis Software and Systems VII, ed. R. Albrecht, R. N. Hook, \& H. A. Bushouse (San Francisco: ASP), ASP Conf. Ser., 145, 108

Skillman, E. D., \& Kennicutt, R. C., Jr. 1993, ApJ, 411, 655

Stasińska, G., \& Schaerer, D. 1999, A\&A, 351, 72

Steidel, C. C., Bogosavljević, M., Shapley, A. E., et al. 2011, ApJ, 736, 160

Stetson, P. B. 1979, PASP, 99, 191

Stockton, A., McGrath, E., Ganalizo, G., Iye, M., \& Maihara, T. 2008, ApJ, 672, 146

Terlevich, R., Melnick, J., Masegosa, J., Moles, M., \& Copetti, M. V. F. 1991, A\&AS, 91, 285

Tikhonov, N. A. 2007, Astron. Lett., 33, 3

Tosi, M., Sabbi, E., Bellazzini, M., et al. 2001, AJ, 122, 1271

Tosi, M., Aloisi, A., Mack, J., Maio, M. 2007, in Galaxy Evolution Across the Hubble Time, ed. F. Combes, \& J. Palous, IAU Symp., 235, 65

van Dokkum, P. G., Whitaker, K. E., Brammer, G., et al. 2010, ApJ, 709, 1018

van Zee, L. 2000, AJ, 119, 2757

van Zee, L., Westphahl, D., Haynes, M., \& Salzer, J. J. 1998, AJ, 115, 1000

Viallefond, F., Lequeux, J., \& Comte, C. 1987, in Starbursts and Galaxy Evolution, ed. T. X. Thuan, T. Montmerle, \& J. Tran Thanh Van (Éditions Frontières), 139

Vílchez, J. M., \& Iglesias-Páramo, J. 1998, ApJ, 508, 248

Villar-Martín, M., Vernet, J., di Serego Alighieri, S., et al. 2003, MNRAS, 346, 273

Weidner, C., \& Kroupa, P. 2005, ApJ, 625, 754

Westmoquette, M. S., Exter, K. M., Smith, L. J., \& Gallagher, J. S. 2007, MNRAS, 381, 894

Yakobchuk, T. M., \& Izotov, Y. I. 2006, Kinematika i Fizika Nebesnykh Tel, 22, 432

Zackrisson, E., Bergvall, N., Olofsson, K., \& Siebert, A. 2001, A\&A, 375, 814

Zackrisson, E., Bergvall, N., Östlin, G., Micheva, G., \& Leksell, M. 2006, ApJ, 650,812

Zackrisson, E., Bergvall, N., \& Leitet, E. 2008, ApJ, 676, L9

Zackrisson, E., Micheva, G., \& Östlin, G. 2009, MNRAS, 397, 2057

Zwicky, F. 1966, ApJ, 143, 192 